\title{
Perturbative reduction of derivative order in EFT
}

\author{
Dražen Glavan \\ Institute of Theoretical Physics, Faculty of Physics, University of Warsaw, \\ Pasteura 5, 02-093 Warsaw, Poland \\ E-mail: drazen.glavan@fuw.edu.pl
}

ABSTRACT: Higher derivative corrections are ubiquitous in effective field theories, which seemingly introduces new degrees of freedom at successive orders. This is actually an artefact of the implicit local derivative expansion defining effective field theories. We argue that higher derivative corrections that introduce additional degrees of freedom should be removed and their effects captured either by lower derivative corrections, or special combinations of higher derivative corrections not propagating extra degrees of freedom. Three methods adapted for this task are examined and field redefinitions are found to be most appropriate. First order higher derivative corrections in a scalar tensor theory are removed by field redefinition and it is found that their effects are captured by a subset of Horndeski theories. A case is made for restricting the effective field theory expansions in principle to only terms not introducing additional degrees of freedom.

KEYwords: Effective Field Theories, Classical Theories of Gravity, Cosmology of Theories beyond the SM

ArXiv EPrint: 1710.01562 


\section{Contents}

1 Introduction 1

2 Method of perturbative constraints $\quad 6$

3 Derivative reduction in single scalar EFT 11

$\begin{array}{ll}3.1 & \text { Derivative reduction at first order } \\ \end{array}$

3.1.1 Reduction at the equation of motion level 13

$\begin{array}{lll}3.1 .2 & \text { Reduction via perturbative constraints } & 14\end{array}$

$\begin{array}{ll}\text { 3.1.3 Derivative reduction via field redefinition } & 16\end{array}$

$\begin{array}{ll}3.2 & \text { Derivative reduction at second order }\end{array}$

$\begin{array}{lll}3.2 .1 & \text { Reduction at the equation of motion level } & 17\end{array}$

$\begin{array}{lll}3.2 .2 & \text { Reduction via perturbative constraints } & 18\end{array}$

$\begin{array}{ll}3.2 .3 & \text { Derivative reduction via field redefinition }\end{array}$

3.3 Derivative reduction at third order - Part I 21

$\begin{array}{lll}3.3 .1 & \text { Reduction at the equation of motion level } & 21\end{array}$

$\begin{array}{lll}3.3 .2 & \text { Reduction via perturbative constraints } & 22\end{array}$

3.3.3 Reduction via field redefinition 24

3.4 Derivative reduction at third order - Part II 24

3.5 Summary of the single scalar case study 25

4 Derivative reduction in scalar-tensor theory 26

5 Conclusions $\quad 30$

\section{Introduction}

Effective theories are a physical framework based on an observation that not all scales of nature are relevant for describing of a particular given system [1-3]. Usually it pertains to descriptions of some low energy phenomenon in terms of the degrees of freedom and interactions relevant for that system, with typical energy scales well below some higher scales typical for the interactions and degrees of freedom which we neglect. It is not to say that we have to neglect the effect of physics on higher scales entirely. We may study the small corrections that it supplies to our low energy system, but we do not need to know exactly what that physics is. Its effects can be captured in terms of the degrees of freedom of the low energy system by introducing additional effective interactions suppressed by the ratio of scales typical for the system at hand and the high scale of new physics. In field theories such effects are encoded by all the possible corrections to the leading order action organized as an expansion in the inverse powers of the high energy (or mass) scale. At each order all that is demanded from the correction terms is to satisfy the assumed symmetries and to have the right dimensionality. 
Higher derivative corrections in effective field theory (EFT) are ubiquitous, and are very hard to avoid. Such corrections, taken at face value, tend to exhibit unphysical behaviour. The issues regarding them were most recently discussed by Burgess and Williams [4], who also argued we typically need not worry about the said issues. Here we make a case for the issues actually not being there if one treats higher derivatives in EFTs correctly in the same perturbative spirit in which they arise in the first place. The same point of view is taken by Solomon and Trodden in [5], who addresse the same types of issues addressed in this work

It is known that higher derivative theories in general exhibit unphysical behavior. The most well known issue encountered is that of Ostrogradsky instabilities [6, 7]. Higher derivative theories generally contain more degrees of freedom per dynamical variable as compared to the lower derivative theories, and such theories we refer to as genuine higher derivative theories in this paper. Usually these extra degrees of freedom are ghost-like, and their excitation can lower the energy of the system without bounds. This is always true for theories without constraints, but can in certain cases be avoided in constrained theories (e.g. $f(R)$ gravity). Another issue often exhibited by higher derivative theories is the existence of runaway solutions. It is important to emphasize that there are theories with higher derivatives in the action that are not genuine in the sense defined above, since they contain the same number of degrees of freedom as their lower order counterparts with the same number of dynamical variables. Examples of such non-genuine higher derivative theories are the Horndeski theories [8], or equivalently generalized Galileons [9-11], and more generally the beyond Horndeski theories [12, 13], and all the DHOST theories [14-16], which are defined as the most general scalar-tensor theories with three propagating degrees of freedom. These are studied very much today in the context of the effective field theory of dark energy $[17,18]$. For convenience, we sometimes refer to non-genuine higher derivative theories as lower derivative theories when it is obvious we are emphasizing their property of not carrying extra degrees of freedom.

Effective field theories in actuality are not genuine higher derivative theories. This becomes clear when we examine cases where we can actually derive them from a more fundamental theory by integrating out some heavy degrees of freedom. In principle, we do not need to assume much about the degrees of freedom being integrated out. This procedure produces an effective action for the system which contains nonlocal terms which do not carry extra degrees of freedom. By construction, the procedure of integrating out degrees of freedom can only lower their total number in the resulting theory. Only when we decide that the degrees of freedom integrated out are heavy with respect to some energy scale we are interested in, can the nonlocal terms in the action be considered small, and expanded in inverse powers of the heavy mass scale. This expansion corresponds to a local derivative expansion of the nonlocal terms, and this is how higher derivative terms appear in the low energy EFT. The modern approach to field theories is to consider them as EFTs up to some higher energy scale. Then naturally all field theories can be considered to have corrections suppressed by some high energy (mass) scale. But here we maintain that these corrections ought to descend from some in principle nonlocal corrections. 
At successively higher orders in EFTs terms with higher and higher derivatives appear. It makes no physical sense that each order of perturbation theory introduces new degrees of freedom. Rather, this is an artifact of our approximation scheme. The observation that the higher derivative terms appear perturbatively, and that their effects should be taken into account perturbatively as well, resolves the issue of extra degrees of freedom. They never appear in perturbatively expandable solutions.

This problem was first encountered and studied in the context of Abraham-Lorentz theory of the electron [19], where the third time derivative of the electron's position appears in the equation of motion. This third derivative is there to take into account the electron's reaction to its own radiation. The space of solutions of this theory contains some clearly unphysical runaway solutions - one can set initial conditions for the electron to be at rest, and it can still accelerate to velocities arbitrarily close to the speed of light. This is an example of the runaway solution due to equations of motion requiring the specification of the initial acceleration in addition to the initial position and velocity. It is an unphysical behavior introduced by treating the higher derivative corrections on the same footing as the leading order equation. One must discard all the solutions of the equations of motion that are not analytic in the expansion parameter as unphysical.

The aim of this paper is to investigate three different methods of correctly accounting for the higher derivative terms in the EFT expansion. They all aim to reduce the derivative order of the higher derivative corrections, representing to them in terms on lower derivative ones, and hence we refer them as derivative reduction methods. In particular we consider (i) derivative reduction on the level of the equations of motion, (ii) derivative reduction by method of perturbative constraints (described in section 2), and (iii) derivative reduction via field redefinition. Their advantages and disadvantages, utility and drawbacks are analyzed in detail in section 3 on a series of examples. They all serve to motivate a the question about the nature of the EFT expansion that we wish to examine: When are genuine higher derivative corrections appearing in EFTs completely captured by the most general corrections not introducing extra degrees of freedom?

It is common lore, mostly due to the work of Simon and Parker [20,21], that the higher derivative terms can always be removed from the equations of motion by perturbatively applying lower order equations of motion. This is certainly true for any single particle systems, such as the minisuperspace model they considered, where the only dynamical variable is the scale factor (see also [22]). Even though the study of this single particle models are insightful, multiple issues and subtleties of this derivative reduction method are missed. One of the things that we examine in this paper is the analogous derivative reduction procedure at the equation of motion level in the field theory system possessing Lorentz symmetries, and whether this derivative reduction procedure is consistent with maintaining Lorentz symmetry. Our findings are that in general it is not, without allowing for field redefinitions. The same conclusion ought to be valid when considering local symmetries, such as in the EFT of gravity.

Reduction of the derivative order at the level of the equations of motion (if needed supplemented by field redefinition) is sufficient if one is interested in obtaining solutions as power series in the expansion parameter truncated to a given order (for some applications 
of this strategy to EFT of gravity see $[23,24])$. Sometimes such solutions are not sufficient. This is often the case in nonequilibrium systems [25], where spurious secular phenomena might be introduced by considering perturbative corrections to the leading order solutions. In such cases a resummation scheme is called for, which, in broad strokes, corresponds to solving exactly the truncated EFT equation with reduced derivatives. For instance, such circumstances arise when studying cosmological solutions in general relativity with EFT corrections. The problem that arises here is that the derivative reduction at the level of the equations of motion is not unique, in the sense that multiple equations accomplish the same task of capturing perturbative solutions to a given order. Attempting the resummed solution for different equations might lead to rather different results, and even exhibit effects such as energy non-conservation in conservative systems. ${ }^{1}$ The correct way to avoid such spurious effects is to base the resummed solutions on the equations of motion that derive from an action principle, which guarantees properties such as energy conservation to all orders. The consideration of nonequilibrium systems motivates us to develop a formalism for reducing the derivative order of EFT corrections directly on the level of the action. Most of this work is devoted to the investigation of such methods.

Higher derivative EFT corrections are particularly awkward if one wishes to consider them in the canonical (Hamiltonian) formalism. The initial motivation for this work came from the problem of constructing and quantizing higher order gauge-invariant cosmological perturbations $^{2}$ in a single scalar inflationary model [26-28], with the idea of addressing certain issues regarding the loop correction to the primordial power spectrum (see [29] and references therein). The formalism most suited for finding the gauge-invariant perturbations seems to be the canonical one, since it makes the constraint structure of the theory explicit [30]. The quantization of these higher order perturbations necessarily requires the introduction of higher derivative terms to the action in order to guarantee one-loop renormalizability [31]. But these higher derivative terms alter the structure of cosmological perturbations beyond linear order, and should be considered right from the start. Hence we need to consider these higher derivative terms in the canonical formalism.

In the canonical formalism every degree of freedom is promoted to an independent field, including the degrees of freedom associated to higher derivative terms in the EFT expansion [32]. In the EFTs this is very cumbersome, since the resulting Hamiltonian is no longer perturbative in the inverse powers of the heavy mass scale assumed to be the control parameter in the EFT expansion, but rather has terms containing positive powers of this mass parameter. This is clearly a signal of doing something wrong. In fact, it means precisely what we have stated, considering higher derivative terms to introduce extra degrees of freedom is inconsistent with the suppositions of EFT.

The very derivation of the canonical formalism in the presence of higher derivative EFT corrections has to be modified to accommodate for the requirement of perturbativity

\footnotetext{
${ }^{1}$ This point is understood in the context of the $2 \mathrm{PI}$ resummation scheme in QFT. By consistently truncating the Dyson-Schwinger equations it is possible to derive a family of equations that are all equivalent to the 2PI equation to the given order, but only one of them corresponds to the 2PI one which can be derived from an action principle. It is said that the other equations overcount the diagrams.

${ }^{2}$ Higher order here refers to higher order in infinitesimal diffeomorphism transformations.
} 
in the expansion parameter. This was noted and clarified by Jaen, Llosa, and Molina, [34], and by Eliezer and Woodard [35]. The perturbativity requirement effectively introduces perturbative second-class constraints into the theory [33], which when solved for reduce the total number of degrees of freedom to the correct one, resulting in the lower order phase space action. Section 2 is devoted to recasting this method in a somewhat different form, and to the discussion of its possible drawbacks. Such a method would be preferable as a way to reconcile higher derivative corrections with the physical assumptions EFT expansion makes, since it would automatically mean that perturbativity requirement maps higher derivative EFT corrections onto lower derivative EFT ones. Larger part of section 3 is devoted to applying this method to a scalar field system, where it is shown that even though the method always accomplishes what it is designed to do - produces a lower derivative phase space action - it in general does not provide a configuration space action (the Lagrangian) which makes it rather impractical for applications.

The third method of derivative reduction that we consider in this work is the field redefinition method. It ought be true that the physics (whatever is measured in the experiments) should not be altered by one's choice of field variables describing the system. Hence, it is in principle permissible to perform transformations from one set of field variables to another (field redefinitions). There is a physical requirement on these redefinitions that they should not change the number of degrees of freedom system. Considering derivatives of the field, it means they should not contain any. In other words, we are not allowed to make a higher derivative theory out of the lower derivative one.

Consider now field redefinitions is the EFTs. Assume that we know the exact form of the nonlocal correction to the action, before it is approximated by a local derivative expansion. Now we can imagine making a nonlocal field redefinition in such an action, resulting in another nonlocal action. We can assume this field redefinition to be a special one - by requiring that the resulting nonlocal action has a local derivative expansion that contains no genuine higher derivative terms. Such special nonlocal transformation would thus eliminates spurious degrees of freedom from the low energy EFT. Now consider taking a short-cut of this construction, directly on the low energy EFT. It is equivalent to making a local field redefinition containing derivatives, that removes all the genuine higher derivative terms from the EFT expansion to a given order. It is due to these considerations that field redefinitions containing derivatives are allowed in EFTs, in the sense that field redefinitions themselves are treated as local derivative expansions. It is found in this paper that the field redefinition method of the derivative reduction is in general superior to the remaining two, especially when considering symmetries of the field systems.

The three mentioned methods for reducing the derivative order of EFT corrections are studied in this paper on several examples of a single scalar field in flat space. Attention is paid to the compatibility of these methods with Lorentz symmetry, and the possibility of obtaining a configuration space (Lagrangian) formulation of the reduced system. Field redefinitions are found to be the preferable method. The field redefinition method is then applied to the first order EFT corrections of a scalar-tensor theory, where it is found that the corrections are captured by the subset of Horndeski actions, which are the most general scalar-theories possessing second order equations of motion. In [5] the derivative reduction 
on the level of the equations of motion, and derivative reduction by field redefinitions are studied on similar examples, and the findings agree with ones presented here.

The introductory section that is drawing to a close serves to define and motivate the study of higher derivatives in the context of effective field theories. Section 2 is devoted to the presentation of the method of derivative reduction via perturbative constraints. In section 3 we apply the three derivative reduction methods to four cases of a single scalar field with higher derivative corrections. The lessons learned from these examples are summarized at the end of section 3. In section 4 the field redefinition method is employed to eliminate the first order higher derivative corrections from the EFT expansion of a scalar-tensor theory. The last section contains the discussion of the results and the concluding remarks.

\section{Method of perturbative constraints}

This section presents the method of perturbative derivative reduction by implementing perturbative constraints. ${ }^{3}$ The method has been developed for systems without constraints by Jaén, Molina and Llosa [34], and by Eliezer and Woodard [35]. Here we present a modified version of it, which has a few advantages: (i) it applies to systems with arbitrary leading order actions, not only to the case of a free leading order system, and (ii) it applies to explicitly time dependent systems, and ones where Noether current is cumbersome to define, (iii) it is easily generalized to systems with constraints.

Even though in this work we aim to investigate field systems, the method here is presented for a multi-particle systems. The reason is that the notation is simpler, and we do not have to explicitly write gradients, which just obscures the expressions. Nevertheless, the generalization to field systems is obvious and straightforward.

Throughout this section, and later when applying the method we use Dirac's notation for equalities [33], where we distinguish between strong equality (off-shell equality) "=" denotes the equality which is true on the level of the action, without applying the equations of motions, and a weak equality (on-shell equality) " $\approx$ " designating equalities valid only after the imposition of equations of motion.

Say that we are interested in studying a system of particles described by variables $x_{i}$, that at leading order is specified by the leading order action,

$$
S^{(0)}\left[x_{i}\right]=\int d t \mathscr{L}^{(0)}\left(x_{i}, \dot{x}_{i}\right),
$$

which is assumed not to be singular, i.e. its Hessian is supposed invertible,

$$
\operatorname{det}\left(\frac{\partial^{2} \mathscr{L}^{(0)}}{\partial \dot{x}_{i} \partial \dot{x}_{j}}\right) \neq 0,
$$

and there are no constraints. The generalization of this method to systems with constraints is beyond the scope of this paper. We consider the first $N$ corrections to this action in the

\footnotetext{
${ }^{3}$ Dubbed localization by the authors of [35].
} 
form of an EFT expansion in terms (operators) of successively higher dimension, with $\epsilon$ being the expansion parameter, given by the configuration space action,

$$
S\left[x_{i}\right]=S^{(0)}\left[x_{i}\right]+\epsilon S^{(1)}\left[x_{i}\right]+\epsilon^{2} S^{(2)}\left[x_{i}\right]+\cdots+\epsilon^{N} S^{(N)}\left[x_{i}\right]=\sum_{n=0}^{N} \epsilon^{n} S^{(n)}\left[x_{i}\right] .
$$

The higher order corrections are also assumed to contain irreducible higher time derivatives, ${ }^{4}$ with each order introducing one more derivative,

$$
S^{(n)}\left[x_{i}\right]=\int d t \mathscr{L}^{(n)}\left(x_{i}, \dot{x}_{i}, \ldots, x_{i}^{[n]}, x_{i}^{[n+1]}\right), \quad x_{i}^{[m]} \equiv \frac{d^{m}}{d t^{m}} x_{i},
$$

so that each new order introduces one derivative more. In what follows we construct a method to isolate a subspace of solutions of this theory that are regular in the expansion parameter $\epsilon$, i.e. that are perturbatively expandable in $\epsilon$.

The starting point of the method is to define the extended action [32], which can be seen as the intermediate step between the Lagrangian and the Hamiltonian formalism, and is particularly suitable for theories with constraints. The extended action is defined by promoting all the time derivatives to independent velocity variables, $x_{i}^{[n]} \rightarrow V_{i}^{n}$, and introducing Lagrange multipliers $\pi_{i}, \pi_{i}^{1}, \ldots, \pi_{i}^{N-1}$ to ensure the on-shell equivalence of the two formulations,

$$
\begin{aligned}
& \mathcal{S}\left[x_{i}, V_{i}^{1}, V_{i}^{2}, \ldots, V_{i}^{N+1}, \pi_{i}, \pi_{i}^{1}, \ldots, \pi_{i}^{N}\right] \\
&=\int d t\left\{\mathscr{L}^{(0)}\left(x_{i}, V_{i}^{1}\right)+\epsilon \mathscr{L}^{(1)}\left(x_{i}, V_{i}^{i}, V_{i}^{2}\right)+\cdots+\epsilon^{N} \mathscr{L}^{(N)}\left(x_{i}, V_{i}^{1}, \ldots, V_{i}^{N+1}\right)\right. \\
&\left.\quad+\pi_{i}\left(\dot{x}_{i}-V_{i}^{1}\right)+\pi_{i}^{1}\left(\dot{V}_{1}-V_{i}^{2}\right)+\cdots+\pi_{i}^{N}\left(\dot{V}_{i}^{N}-V_{i}^{N+1}\right)\right\}
\end{aligned}
$$

The canonical formalism, namely the phase space action $\mathscr{S}$ is now one step away. One needs to solve for the highest velocities $V_{i}^{N+1}$ from the equation descending from the variation of the extended action with respect to $V_{i}^{N+1}$ 's,

$$
\frac{\delta \mathcal{S}}{\delta V_{i}^{N+1}}=\epsilon^{N} \frac{\partial \mathscr{L}^{(N)}}{\partial V_{i}^{N+1}}-\pi_{i}^{N} \approx 0
$$

which is an algebraic relation involving no time derivatives and plug it back into $\mathcal{S}$. The solutions for $V_{i}^{N}$ 's,

$$
V_{i}^{N+1} \approx \bar{V}_{i}^{N+1}\left(x_{i}, V_{i}^{1}, \ldots V_{i}^{N}, \pi_{i}, \pi_{i}^{1}, \ldots \pi_{i}^{N}\right)
$$

are then plugged back into $\mathcal{S}$ as strong equalities, which results in the phase space action,

$$
\begin{aligned}
\mathscr{S}\left[x_{i},\right. & \left.\pi_{i}, V_{i}^{1}, \pi_{i}^{1}, \ldots, V_{i}^{N}, \pi_{i}^{N}\right] \\
& =\int d t\left\{\pi_{i} \dot{x}_{i}+\pi_{i}^{1} \dot{V}_{i}^{1}+\cdots+\pi_{i}^{N} \dot{V}_{i}^{N}-H\left(x_{i}, \pi_{i}, V_{i}^{1}, \pi_{i}^{1}, \ldots V_{i}^{N}, \pi_{i}^{N}\right)\right\},
\end{aligned}
$$

\footnotetext{
${ }^{4}$ Higher time derivatives that can be removed by partial integration are considered reducible.
} 
where the Hamiltonian is

$$
\begin{aligned}
H= & \pi_{i} V_{i}^{1}+\pi_{i}^{1} V_{i}^{2}+\cdots+\pi_{i}^{N-1} V_{i}^{N}+\pi^{N} \bar{V}_{i}^{N+1} \\
& -\mathscr{L}^{(0)}\left(x_{i}, V_{i}^{1}\right)-\epsilon \mathscr{L}^{(1)}\left(x_{i}, V_{i}^{1}, V_{i}^{2}\right)-\cdots-\epsilon^{N} \mathscr{L}^{(N)}\left(x_{i}, V_{i}^{1}, V_{i}^{2}, \ldots, \bar{V}_{i}^{N+1}\right) .
\end{aligned}
$$

This phase space action is on-shell equivalent to the extended action (2.5), and hence to the starting configuration space action (2.3) as well. This construction is equivalent to the one introduced by Ostrogradsky $[6,7]$. There is nothing about this construction specific to higher derivative theories. It is perfectly applicable to standard lower derivative theories, and in fact allows for a much more pedagogical introduction of the canonical (Hamiltonian) formalism.

The obstruction to the construction of the phase space action in the case at hand is that equation (2.6) does not admit solutions for $V_{i}^{N+1}$ 's analytic in the perturbative expansion parameter $\epsilon$, and is therefore not admissible by our assumptions. ${ }^{5}$ Given such assumptions, the equation (2.6) actually represents a constraint, and should be treated as such. This observation allows us to isolate from the full theory only the subset of solutions perturbatively expandable in $\epsilon$.

In the language of Dirac's canonical formalism [33] equation (2.6) is a primary constraint. The requirement that this constraint is consistent, i.e. that its time derivative vanishes on-shell produces a secondary constraint. Again requiring consistency, and repeating this process generates a set of constraints that actually end up being second-class, meaning that in the end the variables $V_{i}^{N+1}$ 's are uniquely determined (by the equations of motion). Even though we can cast the whole method in the form usually employed in canonical formalism, by defining Poisson brackets, and working with the Hamiltonian, it is actually not the most practical way to proceed here. It is more convenient to work with the extended action (2.5), and the equations of motion descending from varying it with respect to independent variables.

The systematic way to proceed is to write down all the equations descending from varying the extended action,

$$
\begin{array}{cll}
\frac{\delta \mathcal{S}}{\delta V_{i}^{N+1}} \approx 0 & \Rightarrow & \pi_{i}^{N} \approx \epsilon^{N} \frac{\partial \mathscr{L}^{(N)}}{\partial V_{i}^{N+1}} \\
\frac{\delta \mathcal{S}}{\delta V_{i}^{N}} \approx 0 & \Rightarrow & \dot{\pi}_{i}^{N} \approx-\pi_{i}^{N-1}+\epsilon^{N} \frac{\partial \mathscr{L}^{(N)}}{\partial V_{i}^{N}}+\epsilon^{N-1} \frac{\partial \mathscr{L}^{(N-1)}}{\partial V_{i}^{N}} \\
\vdots & & \\
\frac{\delta \mathcal{S}}{\delta V_{i}^{2}} \approx 0 & \Rightarrow & \dot{\pi}_{i}^{2} \approx-\pi_{i}^{1}+\epsilon^{N} \frac{\partial \mathscr{L}^{(N)}}{\partial V_{i}^{2}}+\cdots+\epsilon^{1} \frac{\partial \mathscr{L}^{(1)}}{\partial V_{i}^{2}} \\
\frac{\delta \mathcal{S}}{\delta V_{i}^{1}} \approx 0 & \Rightarrow & \dot{\pi}_{i}^{1} \approx-\pi_{i}+\epsilon^{N} \frac{\partial \mathscr{L}^{(N)}}{\partial V_{i}^{1}}+\cdots+\epsilon^{0} \frac{\partial \mathscr{L}^{(0)}}{\partial V_{i}^{1}} \\
\frac{\delta \mathcal{S}}{\delta x_{i}} \approx 0 & \Rightarrow & \dot{\pi}_{i} \approx \epsilon^{N} \frac{\partial \mathscr{L}^{(N)}}{\partial x_{i}}+\cdots+\epsilon^{0} \frac{\partial \mathscr{L}^{(0)}}{\partial x_{i}}
\end{array}
$$

\footnotetext{
${ }^{5}$ As the authors of [34] put it, the variables we are dealing with are a quotient $\operatorname{ring} \mathbb{R} / \epsilon^{N+1}$, i.e. polynomials in $\epsilon$ with real coefficients, modulo $\epsilon^{N+1}$.
} 


$$
\begin{array}{cll}
\frac{\delta \mathcal{S}}{\delta \pi_{i}} \approx 0 & \Rightarrow & \dot{x}_{i} \approx V_{i}^{1}, \\
\frac{\delta \mathcal{S}}{\delta \pi_{i}^{1}} \approx 0 & \Rightarrow & \dot{V}_{i}^{1} \approx V_{i}^{2}, \\
\vdots & & \\
\frac{\delta \mathcal{S}}{\delta \pi_{i}^{N}} \approx 0 & &
\end{array}
$$

These equations need to be satisfied at each order in $\epsilon$. Therefore, we may solve them order by order. Multiplying all of the equations by $\epsilon^{N}$ yields the leading order equations,

$$
\begin{aligned}
& \epsilon^{N} \pi_{i}^{N} \approx 0 \\
& \epsilon^{N} \dot{\pi}_{i}^{N} \approx-\epsilon^{N} \\
& \vdots \\
& \epsilon^{N} \dot{\pi}_{i}^{1} \approx-\epsilon^{N} \pi_{i}, \\
& \epsilon^{N} \dot{\pi}_{i} \approx \epsilon^{N} \frac{\partial \mathscr{L}^{(0)}}{\partial x_{i}} \\
& \epsilon^{N} \dot{x}_{i} \approx \epsilon^{N} V_{i}^{N} \\
& \vdots \\
& \epsilon^{N} \dot{V}_{i}^{N}
\end{aligned}
$$

The first equation (2.18) is a constraint. Taking derivative of it generates another constraint. Taking derivative of that one generates yet another one, etc. The result of this process are leading order solutions of the constraints,

$$
\epsilon^{N} \pi_{i}^{n} \approx \epsilon^{N} \bar{\pi}_{i}^{n}\left(x_{i}, \pi_{i}\right), \quad \epsilon^{N} V_{i}^{n} \approx \epsilon^{N} \bar{V}_{i}^{n}\left(x_{i}, \pi_{i}\right),
$$

where we have determined just the leading order term in the $\epsilon$ expansion of $\bar{\pi}_{i}^{n}\left(x_{i}, \pi_{i}\right)$ and $\bar{V}_{i}^{n}\left(x_{i}, \pi_{i}\right)$.

Next step is to multiply equations (2.10)-(2.17) by $\epsilon^{N-1}$ and repeat the same process to obtain

$$
\epsilon^{N-1} \pi_{i}^{n} \approx \epsilon^{N-1} \bar{\pi}_{i}^{n}\left(x_{i}, \pi_{i}\right), \quad \epsilon^{N-1} V_{i}^{n} \approx \epsilon^{N-1} \bar{V}_{i}^{n}\left(x_{i}, \pi_{i}\right),
$$

meaning that we now have first two terms in the $\epsilon$ expansion of $\bar{\pi}_{i}^{n}\left(x_{i}, \pi_{i}\right)$ and $\bar{V}_{i}^{n}\left(x_{i}, \pi_{i}\right)$.

This is repeated until we have solved for all the constraints to obtain

$$
\pi_{i}^{n} \approx \bar{\pi}_{i}^{n}\left(x_{i}, \pi_{i}\right), \quad V_{i}^{n} \approx \bar{V}_{i}^{n}\left(x_{i}, \pi_{i}\right)
$$

to order $\epsilon^{N}$. The final step involves plugging these of-shell relations as strong equalities into the extended action (2.5). This produces the reduced phase space action,

$$
\mathscr{S}_{\text {red }}\left[x_{i}, \pi_{i}\right]=\int d^{D} x\left\{\pi_{i} \dot{x}_{1}+\bar{\pi}_{i}^{1} \dot{\bar{V}}_{i}^{1}+\cdots+\bar{\pi}_{1}^{N} \dot{\bar{V}}_{i}^{N}-H_{\text {red }}\left(x_{i}, \pi_{i}\right)\right\},
$$


where the reduced Hamiltonian is

$$
\begin{aligned}
H_{\mathrm{red}}\left(x_{i}, \pi_{i}\right)= & \bar{\pi}_{i} \bar{V}_{i}^{1}+\bar{\pi}_{i}^{1} \bar{V}_{i}^{1}+\cdots+\bar{\pi}_{i}^{N} \bar{V}_{i}^{N+1}-\mathscr{L}^{(0)}\left(x_{i}, \bar{V}_{i}^{1}\right) \\
& -\epsilon \mathscr{L}^{(1)}\left(x_{i}, \bar{V}_{i}^{1}, \bar{V}_{i}^{2}\right)-\cdots-\epsilon^{N} \mathscr{L}^{(N)}\left(x_{i}, \bar{V}_{i}^{1}, \bar{V}_{i}^{2}, \ldots, V_{i}^{N}\right) .
\end{aligned}
$$

This phase space action now depends explicitly only on the degrees of freedom found in the leading order action.

The nonstandard feature of the phase space action (2.27) is the non-canonical kinetic term (the part containing the time derivatives). The reader familiar with Dirac's canonical formalism will recognize this actually defining the Dirac brackets between variables. We are not interested in the brackets here so we will not work them out (for examples of this see [35]). We are interested though whether there is a configutation space equivalent of the reduced phase space action (2.27). The symplectic part plays a crucial role in the answer to this question.

Since $\bar{V}_{i}^{n}$ 's and $\bar{\pi}_{i}^{n}$ 's are functions of $x_{i}$ and $\pi_{i}$ only, the symplectic part of the action can be written as

$$
\int d^{D} x\left\{\left[\pi_{i}+\epsilon \Pi_{i}\left(x_{j}, \pi_{j}\right)\right] \dot{x}_{i}+\epsilon X_{i}\left(x_{j}, \pi_{j}\right) \dot{\pi}_{i}\right\},
$$

where $\Pi_{i}$ and $X_{i}$ have some expansion in $\epsilon$. In general no amount of partial integrations can remove the time derivative from $\pi_{i}$ completely. In such a case one cannot follow the standard procedure to obtain the configuration space action. Usually what one does when there is no $\dot{\pi}_{i}$ is solve for the conjugate momenta from the Hamilton's equations as functions of $x_{i}$ and $\dot{x}_{i}$,

$$
\pi_{i} \approx \bar{\pi}_{i}\left(x_{i}, \dot{x}_{i}\right)
$$

and then plugs this into the phase space action as a strong equality. This then results in the configuration space action, and the Lagrangian formulation. The reason why it works is that it can easily be shown that two formulations are on-shell equivalent. But when $\dot{\pi}_{i}$ is present in the symplectic part of the action in an irreducible way this procedure does not result in the on-shell equivalent configuration space action, meaning there is no Lagrangian formulation of the reduced system only in terms of $x_{i}$ 's. Note that this never happens for systems with a single degree of freedom where $\dot{\pi}$ can always be removed. ${ }^{6}$

One can remove the irreducible $\dot{\pi}_{i}$ 's from the symplectic part by a redefinition of the variables $x_{i}$ 's. Unfortunately this procedure is not unique, and more likely than not one will make a redefinition which for example breaks manifest Lorentz covariance. Even though there is nothing fundamentally wrong with that, this is reason why we shy away from making variable redefinitions in the phase space action, since it is very likely to complicate things. That being said, if one is interested only in the phase space formulation, action (2.27) can always be brought to the canonical form by redefining the variables and their conjugate momenta perturbatively in $\epsilon$.

\footnotetext{
${ }^{6}$ This should not to be confused with systems with a single local degree of freedom, such as the scalar field, which actually has uncountably many degrees of freedom.
} 
The method presented in this section is applied to a few cases involving a single scalar field in the next section. If the reader find the presentation of the method in this section too dense, two examples in the next section are worked out in detail.

\section{Derivative reduction in single scalar EFT}

This section serves to test, examine, and compare the three methods of perturbative derivative reduction on simple examples of real scalar field systems. Note that this is not a one-particle system, but a system with one local degree of freedom, interacting with the neighboring ones via gradients. We work in $D$ spacetime dimensions, with a positive signature Minkowski metric; the D'Alembertian is $\square=\partial_{\mu} \partial^{\mu}$, and the Laplacian $\partial_{i} \partial^{i}$, and a dot denotes a time derivative, while a prime denote a derivative with respect to the field.

We consider an EFT expansion in the system of a single scalar field, up to cubic order in the expansion parameter $\epsilon=M^{-2}$, where $M$ is some heavy mass scale,

$$
S[\phi]=S^{(0)}[\phi]+\epsilon S^{(1)}[\phi]+\epsilon^{2} S^{(2)}[\phi]+\epsilon^{3} S^{(3)}[\phi]+\mathcal{O}\left(\epsilon^{4}\right),
$$

where the leading order action is taken to be a scalar with a canonical kinetic term, and a potential term,

$$
S^{(0)}=\int d^{D} x\left\{-\frac{1}{2}\left(\partial_{\mu} \phi\right)\left(\partial^{\mu} \phi\right)-\mathrm{U}(\phi)\right\} .
$$

The corrections to this action are assumed to be all possible Lorentz invariant terms of certain mass dimension, and the expansion is ordered in the number of these mass dimensions. The first order correction,

$$
\epsilon S^{(1)}=\epsilon \sum_{n=1}^{3} \alpha_{n}^{(1)} S_{n}^{(1)},
$$

is composed out of all possible independent dimension 6 terms (operators),

$$
\begin{aligned}
S_{1}^{(1)} & =\int d^{D} x \phi^{6}, \\
S_{2}^{(1)} & =\int d^{D} x \phi^{2}\left(\partial_{\mu} \phi\right)\left(\partial^{\mu} \phi\right), \\
S_{3}^{(1)} & =\int d^{D} x(\square \phi)(\square \phi),
\end{aligned}
$$

the second order correction,

$$
\epsilon^{2} S^{(2)}=\epsilon^{2} \sum_{n=1}^{6} \alpha_{n}^{(2)} S_{n}^{(2)},
$$

out of all possible independent dimension 8 terms,

$$
\begin{aligned}
& S_{1}^{(2)}=\int d^{D} x \phi^{8}, \\
& S_{2}^{(2)}=\int d^{D} x \phi^{4}\left(\partial_{\mu} \phi\right)\left(\partial^{\mu} \phi\right),
\end{aligned}
$$




$$
\begin{aligned}
S_{3}^{(2)} & =\int d^{D} x\left(\partial_{\mu} \phi\right)\left(\partial^{\mu} \phi\right)\left(\partial_{\nu} \phi\right)\left(\partial^{\nu} \phi\right), \\
S_{4}^{(2)} & =\int d^{D} x \phi\left(\partial_{\mu} \phi\right)\left(\partial^{\mu} \phi\right)(\square \phi), \\
S_{5}^{(2)} & =\int d^{D} x \phi^{2}(\square \phi)(\square \phi), \\
S_{6}^{(2)} & =\int d^{D} x\left(\partial_{\mu} \square \phi\right)\left(\partial^{\mu} \square \phi\right),
\end{aligned}
$$

and the third order correction,

$$
\epsilon^{3} S^{(3)}=\epsilon^{3} \sum_{n=1}^{13} \alpha_{n}^{(3)} S_{n}^{(3)},
$$

out of all possible independent dimension 10 terms,

$$
\begin{aligned}
S_{1}^{(3)} & =\int d^{D} x \phi^{10}, \\
S_{2}^{(3)} & =\int d^{D} x \phi^{6}\left(\partial_{\mu} \phi\right)\left(\partial^{\mu} \phi\right), \\
S_{3}^{(3)} & =\int d^{D} x \phi^{2}\left(\partial_{\mu} \phi\right)\left(\partial^{\mu} \phi\right)\left(\partial_{\nu} \phi\right)\left(\partial^{\nu} \phi\right), \\
S_{4}^{(3)} & =\int d^{D} x \phi^{3}\left(\partial_{\mu} \phi\right)\left(\partial^{\mu} \phi\right)(\square \phi), \\
S_{5}^{(3)} & =\int d^{D} x \phi^{4}(\square \phi)(\square \phi), \\
S_{6}^{(3)} & =\int d^{D} x\left(\partial_{\mu} \phi\right)\left(\partial^{\mu} \phi\right)(\square \phi)(\square \phi), \\
S_{7}^{(3)} & =\int d^{D} x\left(\partial_{\mu} \phi\right)\left(\partial^{\mu} \phi\right)\left(\partial_{\nu} \partial_{\rho} \phi\right)\left(\partial^{\nu} \partial^{\rho} \phi\right), \\
S_{8}^{(3)} & =\int d^{D} x\left(\partial_{\mu} \phi\right)\left(\partial_{\nu} \phi\right)\left(\partial^{\mu} \partial^{\nu} \phi\right)(\square \phi), \\
S_{9}^{(3)} & =\int d^{D} x \phi(\square \phi)(\square \phi)(\square \phi), \\
S_{10}^{(3)} & =\int d^{D} x \phi\left(\partial_{\mu} \partial_{\nu} \phi\right)\left(\partial^{\mu} \partial^{\nu} \phi\right)(\square \phi), \\
S_{11}^{(3)} & =\int d^{D} x \phi\left(\partial_{\mu} \partial_{\nu} \phi\right)\left(\partial^{\nu} \partial^{\rho} \phi\right)\left(\partial_{\rho} \partial^{\mu} \phi\right), \\
S_{12}^{(3)} & =\int d^{D} x \phi^{2}\left(\partial_{\mu} \square \phi\right)\left(\partial^{\mu} \square \phi\right), \\
S_{13}^{(3)} & =\int d^{D} x(\square \square \phi)(\square \square \phi) .
\end{aligned}
$$

The EFT expansion introduces one higher derivative term (3.6) at linear order, three higher derivative terms (3.11)-(3.13) at second order, and ten (3.18)-(3.27) at third order. This is a rather generic feature of the EFT expansion, and it is hard to come up with a symmetry principle that would exclude them. These higher derivative terms do not mean 
that at each order in $\epsilon$ new degrees of freewhatdom appear. It is rather the assumed approximation scheme that represents corrections as local terms that is to blame for the seeming introduction of extra degrees of freedom. These degrees are spurious, and ought to be treated in the same spirit in which they arise - perturbatively, which actually eliminates them.

The following subsections analyze the reduction of derivative order at successive orders in the expansion parameter $\epsilon$ using three methods - reduction at the equation of motion level, reduction via perturbative constraints method of section 2 , and reduction via field redefinitions. Considering all the possible corrections (3.4)-(3.27) in is not necessary for the points that we want to make, and would only obscure them with the sheer number of formulas without introducing anything essentially new to the discussion. Rather we will only consider the highest derivative term at each order (3.6), (3.13), and (3.27), and in addition (3.21). The emphasis in this section is not on the exact form of the reduced theories, but rather on the lessons that can be learned about methods from considering simpler examples.

It is worth noting that in [36] the Lorentz invariant systems of multiple interacting scalar fields with actions containing irreducible second derivatives were analysed, and classified according whether they can be brought to explicit lower order form, and whether this preserves manifest Lorentz covariance.

\subsection{Derivative reduction at first order}

Let us consider the action (3.2) with the first order correction (3.6),

$$
S=\int d^{D} x\left\{-\frac{1}{2}\left(\partial_{\mu} \phi\right)\left(\partial^{\mu} \phi\right)-U+\epsilon \alpha_{1}(\square \phi)(\square \phi)\right\},
$$

while neglecting all the contributions of order $\mathcal{O}\left(\epsilon^{2}\right)$. We will be looking to reduce the derivative order of this system, and represent it with an equivalent lower derivative system that captures correctly the solutions to order $\epsilon$. Three methods are considered in the following: reducing the derivative order at the level of the equation of motion, reducing the derivative order by implementing perturbative second class constraints of section 2 , and reducing the order via field redefinition.

\subsubsection{Reduction at the equation of motion level}

The equation of motion descending from the action (3.28) is

$$
\square \phi-U^{\prime}+2 \epsilon \alpha_{1} \square \square \phi=0
$$

Applying the leading order equation of motion to the first order term is straightforward and yields

$$
\square \phi-U^{\prime}+2 \epsilon \alpha_{1}\left[U^{\prime \prime} \square \phi+U^{\prime \prime \prime}\left(\partial_{\mu} \phi\right)\left(\partial^{\mu} \phi\right)\right]=0,
$$

which correctly reproduces the perturbative solutions of the original equation (3.29) to linear order in $\epsilon$. But this is not the only lower (time) derivative equation that does it. In 
fact, since we can always use $\square \phi=U^{\prime}$ at linear order, there is a one-parameter family of equations that accomplish this task,

$$
\square \phi-U^{\prime}+2 \epsilon \alpha_{1}\left[(1+b) U^{\prime \prime} \square \phi+U^{\prime \prime \prime}\left(\partial_{\mu} \phi\right)\left(\partial^{\mu} \phi\right)-b U^{\prime \prime} U^{\prime}\right]=0,
$$

where $b$ is the free parameter. There is one distinguished choice for this parameter $b=1$, for which there is an action formulation of the equation of motion, in the sense that the equation following from the action is exactly the one for $b=1$ with no further manipulations required. We will derive this action in the following subsection via the method of perturbative second-class constraints. As noted in the Introduction, an action formulation allows for resummation that conserves exactly the charges associated to global symmetries.

\subsubsection{Reduction via perturbative constraints}

Here we apply the method described in section 2 to reduce the derivative order of the action (3.28). The starting point is the definition of the extended action, which is obtained from the configuration space one by promoting time derivatives to independent fields,

$$
\dot{\phi} \rightarrow V_{1}, \quad-\square \phi=\ddot{\phi}-\nabla^{2} \phi=\dot{V}_{1}-\nabla^{2} \phi \rightarrow V_{2},
$$

and by introducing appropriate Lagrange multipliers $\pi$ and $\pi_{1}$ to ensure the on-shell equivalence,

$$
\begin{aligned}
\mathcal{S} & =\int d^{D} x\left\{\frac{1}{2} V_{1}^{2}-\frac{1}{2}(\nabla \phi)^{2}-U+\epsilon \alpha_{1} V_{2}^{2}+\pi\left(\dot{\phi}-V_{1}\right)+\pi_{1}\left(\dot{V}_{1}-\nabla^{2} \phi-V_{2}\right)\right\} \\
& =\int d^{D} x\left\{\pi \dot{\phi}+\pi_{1} \dot{V}_{1}-\left[\pi V_{1}+\pi_{1}\left(V_{2}+\nabla^{2} \phi\right)-\frac{1}{2} V_{1}^{2}+\frac{1}{2}(\nabla \phi)^{2}+U-\epsilon \alpha_{1} V_{2}^{2}\right]\right\} .
\end{aligned}
$$

Proceeding with the recipe given in section 2, the equations resulting from the action principle with respect to all the independent fields are

$$
\begin{array}{lll}
\frac{\delta \mathcal{S}}{\delta V_{2}} \approx 0 & \Rightarrow & \pi_{1} \approx 2 \epsilon \alpha_{1} V_{2} \\
\frac{\delta \mathcal{S}}{\delta V_{1}} \approx 0 & \Rightarrow & \dot{\pi}_{1} \approx-\pi+V_{1}, \\
\frac{\delta \mathcal{S}}{\delta \phi} \approx 0 & \Rightarrow & \dot{\pi} \approx \nabla^{2} \phi-U^{\prime}-\nabla^{2} \pi_{1}, \\
\frac{\delta \mathcal{S}}{\delta \pi} \approx 0 & \Rightarrow & \dot{\phi} \approx V_{1}, \\
\frac{\delta \mathcal{S}}{\delta \pi_{1}} \approx 0 & \Rightarrow & \dot{V}_{1} \approx V_{2}+\nabla^{2} \phi .
\end{array}
$$

Multiplying these equations by $\epsilon$ and discarding the terms $\mathcal{O}\left(\epsilon^{2}\right)$ gives us the leading order equations,

$$
\begin{aligned}
\epsilon \pi_{1} & \approx 0, \\
\epsilon \dot{\pi}_{1} & \approx \epsilon\left(-\pi+V_{1}\right), \\
\epsilon \dot{\pi} & \approx \epsilon\left(\nabla^{2} \phi-U^{\prime}-\nabla^{2} \pi_{1}\right), \\
\epsilon \dot{\phi} & \approx \epsilon V_{1}, \\
\epsilon \dot{V}_{1} & \approx \epsilon\left(V_{2}+\nabla^{2} \phi\right),
\end{aligned}
$$


where now it is clear that eq. (3.39) takes the form of a primary constraint, and $V_{2}$ is its associated Lagrange multiplier. Requiring the consistency of the primary constraint (vanishing of its time derivative) generates a secondary constraint

$$
\epsilon \dot{\pi}_{1} \approx \epsilon\left(-\pi+V_{1}\right) \approx 0 \quad \Rightarrow \quad \epsilon V_{1} \approx \epsilon \pi .
$$

The two constraint are second-class, and determine the Lagrange multiplier of the primary constraint,

$$
\epsilon \frac{d}{d t}\left(-\pi+V_{1}\right) \approx \epsilon\left(U^{\prime}+V_{2}\right) \approx 0 \quad \Rightarrow \quad \epsilon V_{2} \approx-\epsilon U^{\prime} .
$$

This determines $\pi_{1}, V_{1}, V_{2}$ to leading order depending algebraically of $\phi$ and $\pi$.

After solving for the leading order constraints, we turn to the first order equations (3.39)-(3.43). The first of these equations again takes the form of the primary constraint, because upon using the leading order solution of the constraint equations (3.45) it reads

$$
\pi_{1} \approx-2 \epsilon \alpha_{1} U^{\prime}
$$

In the same way as for the leading order, this primary constraint generates a secondary constraint, the two of them being second-class, and determining the Lagrange multiplier,

$$
\begin{aligned}
& V_{1} \approx \pi-2 \epsilon \alpha_{1} U^{\prime \prime} \pi \\
& V_{2} \approx-U^{\prime}-2 \epsilon \alpha_{1}\left\{U^{\prime \prime \prime}\left[\pi^{2}-(\nabla \phi)^{2}\right]-U^{\prime \prime} U^{\prime}\right\} .
\end{aligned}
$$

We have solved for $\pi_{1}, V_{1}$, and $V_{2}$ to first order as functions of $\pi$ and $\phi$. Since these relations are algebraic, in the sense that they do not involve any time derivatives, we can promote them to strong (off-shell) equalities, and plug them back into the extended action (3.33) to obtain the phase space reduced action, which after a couple of partial integrations reads

$$
\mathscr{S}_{\text {red }}=\int d^{D} x\left\{\left(1+2 \epsilon \alpha_{1} U^{\prime \prime}\right) \pi \dot{\phi}-\left[\frac{1}{2} \pi^{2}+\frac{1}{2}(\nabla \phi)^{2}+U+\epsilon \alpha_{1}\left[2 U^{\prime \prime}(\nabla \phi)^{2}+\left(U^{\prime}\right)^{2}\right]\right]\right\} .
$$

In fact, because of the structure of the extended action, we do not actually need to solve for velocities in this example beyond leading order since their higher orders cancel when plugged into the action.

Note that the phase space action (3.49) obtained via the imposition of perturbative second-class constraints is not in a canonical form, due to the symplectic part not being just $\pi \dot{\phi}$, but is modified by the field dependent prefactor. This modified symplectic part means that the Poissons bracket between $\phi$ and $\pi$ is not canonical, but rather

$$
\left\{\phi(t, \vec{x}), \pi\left(t, \vec{x}^{\prime}\right)\right\}=\frac{\delta^{D-1}\left(\vec{x}-\vec{x}^{\prime}\right)}{1+2 \epsilon \alpha_{1} U^{\prime \prime}}=\left[1-2 \epsilon \alpha_{1} U^{\prime \prime}\right] \delta^{D-1}\left(\vec{x}-\vec{x}^{\prime}\right) .
$$

This is not surprising, since such a bracket results from solving the second-class constraints, and is in fact nothing else then the Dirac bracket.

Even though it is not necessary, the symplectic part of the phase space action can be canonicalized by a non-canonical transformation of the conjugate momentum field,

$$
\pi \rightarrow\left(1-2 \epsilon \alpha_{1} U^{\prime \prime}\right) \pi
$$


after which the phase space action takes the form

$$
\mathscr{S}_{\text {red }}=\int d^{D} x\left\{\pi \dot{\phi}-\left[\frac{1}{2} \pi^{2}+\frac{1}{2}(\nabla \phi)^{2}+U+\epsilon \alpha_{1}\left(2 U^{\prime \prime}\left[-\pi^{2}+(\nabla \phi)^{2}\right]+\left(U^{\prime}\right)^{2}\right)\right]\right\} .
$$

The transformation of the conjugate momentum field (3.51) is not the unique transformation that puts the phase space action into a canonical form. This can also be accomplished by transforming the scalar field itself,

$$
\phi \rightarrow \phi-2 \epsilon \alpha_{1} U^{\prime}
$$

We are hesitant to make field transformations in the phase space action. Even though it is not the case in this specific example, it is very easy to lose manifest Lorentz covariance when making a field transformation that involves gradients of the field, or the conjugate momentum.

The reduced configuration space action is obtained from the phase space one (3.52) in the usual manner. First we solve the Hamilton's equation for the conjugate momentum filed in terms of the scalar and its time derivative,

$$
\frac{\delta \mathscr{S}_{\mathrm{red}}}{\delta \pi} \approx 0 \quad \Rightarrow \quad \pi \approx \dot{\phi}-4 \epsilon \alpha_{1} U^{\prime \prime} \dot{\phi} .
$$

This relation is then promoted to the on-shell equality and plugged into the phase space action to obtain the configuration space one, which can be written in a manifestly Lorentz invariant form

$$
S_{\text {red }}=\int d^{D} x\left\{-\frac{1}{2}\left[1+4 \epsilon \alpha_{1} U^{\prime \prime}\right]\left(\partial_{\mu} \phi\right)\left(\partial^{\mu} \phi\right)-U-\epsilon \alpha_{1}\left(U^{\prime}\right)^{2}\right\} .
$$

The effect of the higher derivative correction when reduced is to renormalize the kinetic term (by a field-dependent factor), and to add another potential term. The equation of motion following from the reduced lower derivative action is

$$
\square \phi-U^{\prime}+2 \epsilon \alpha_{1}\left[2 U^{\prime \prime}(\square \phi)+U^{\prime \prime \prime}\left(\partial_{\mu} \phi\right)\left(\partial^{\mu} \phi\right)-U^{\prime \prime} U^{\prime}\right]
$$

which is precisely the $b=1$ equation (3.31) we have obtained by reducing the derivative order at the level of the equation of motion.

We have derived the lower derivative action (3.55) that captures completely to first order in $\epsilon$ the subspace of perturbatively expandable solutions of the higher derivative action (3.28).

\subsubsection{Derivative reduction via field redefinition}

Since the higher derivative terms in the leading order action (3.28) are assumed to arise perturbatively, and are to be treated perturbatively, we may employ field redefinitions that include higher derivatives. It is simple to see that the following redefinition

$$
\phi \rightarrow \phi-\epsilon \alpha_{1} \square \phi
$$


removes all the higher derivatives to order $\mathcal{O}\left(\epsilon^{2}\right)$,

$$
S \rightarrow \int d^{D} x\left\{-\frac{1}{2}\left[1+2 \alpha_{1} \epsilon U^{\prime \prime}\right]\left(\partial_{\mu} \phi\right)\left(\partial^{\mu} \phi\right)-U\right\}
$$

accomplishing the desired task. This is not the same action obtained by the method of perturbative constraints (3.55). The discrepancy is resolved by noting that here we are still left with the freedom of field redefinitions not including derivatives, which do not change the derivative order of the action. In particular, an additional redefinition,

$$
\phi \rightarrow \phi+\epsilon \alpha_{1} U^{\prime}
$$

transforms the action exactly into (3.55),

$$
S \rightarrow \int d^{D} x\left\{-\frac{1}{2}\left[1+4 \alpha_{1} \epsilon U^{\prime \prime}\right]\left(\partial_{\mu} \phi\right)\left(\partial^{\mu} \phi\right)-U-\epsilon \alpha_{1}\left(U^{\prime}\right)^{2}\right\}
$$

This demonstrates the equivalence of field redefinitions to the other two derivative reduction methods.

\subsection{Derivative reduction at second order}

At the second order in $\epsilon$ we consider only the two higher derivative corrections to the leading order action (3.2), the first one (3.6) arising at linear level, and the second one (3.13) at the quadratic,

$$
S=\int d^{D} x\left\{-\frac{1}{2}\left(\partial_{\mu} \phi\right)\left(\partial^{\mu} \phi\right)-U+\epsilon \alpha_{1}(\square \phi)(\square \phi)+\epsilon^{2} \alpha_{2}\left(\partial_{\mu} \square \phi\right)\left(\partial^{\mu} \square \phi\right)\right\} .
$$

\subsubsection{Reduction at the equation of motion level}

The equation of motion following from the starting higher derivative action is

$$
\square \phi-U^{\prime}+2 \epsilon \alpha_{1} \square \square \phi-2 \epsilon^{2} \alpha_{2} \square \square \square \phi=0 .
$$

By successive applications of the lower order equations in the higher order terms this equation can be reduced to the second order one in derivatives,

$$
\begin{aligned}
\square \phi- & U^{\prime}+2 \epsilon \alpha_{1}\left[U^{\prime \prime} U^{\prime}+U^{\prime \prime \prime}\left(\partial_{\mu} \phi\right)\left(\partial^{\mu} \phi\right)\right]-2 \epsilon^{2}\left(\alpha_{2}+2 \alpha_{1}^{2}\right)\left\{\left[U^{\prime \prime} U^{\prime}\right]^{\prime} U^{\prime}\right. \\
+ & {\left[2 U^{(4)} U^{\prime}+5 U^{\prime \prime \prime} U^{\prime \prime}\right]\left(\partial_{\mu} \phi\right)\left(\partial^{\mu} \phi\right)+U^{(5)}\left(\partial_{\mu} \phi\right)\left(\partial^{\mu} \phi\right)\left(\partial_{\nu} \phi\right)\left(\partial^{\nu} \phi\right) } \\
+ & \left.4 U^{(4)}\left(\partial^{\mu} \phi\right)\left(\partial^{\nu} \phi\right)\left(\partial_{\mu} \partial_{\nu} \phi\right)+2 U^{\prime \prime \prime}\left(\partial_{\mu} \partial_{\nu} \phi\right)\left(\partial^{\mu} \partial^{\nu} \phi\right)\right\}=0 .
\end{aligned}
$$

There is no obstacle to accomplishing this task. Just as in the first order case, this is not a unique equation capturing solutions to the second order in $\epsilon$, and only particular forms of the reduced equation have action formulations. 


\subsubsection{Reduction via perturbative constraints}

The starting point of the method of derivative reduction via perturbative constraints is the definition of the extended action. It is obtained by promoting time derivatives to independent fields,

$$
\dot{\phi} \rightarrow V_{1}, \quad-\square \phi=\dot{V}_{1}-\nabla^{2} \phi \rightarrow V_{2}, \quad-\partial_{0} \square \phi=\dot{V}_{2} \rightarrow V_{3},
$$

and introducing Lagrange multipliers $\pi, \pi_{1}, \pi_{2}$ to ensure on-shell equivalence,

$$
\begin{gathered}
\mathcal{S}=\int d^{D} x\left\{\frac{1}{2} V_{1}^{2}-\frac{1}{2}(\nabla \phi)^{2}-U+\epsilon \alpha_{1} V_{2}^{2}-\epsilon^{2} \alpha_{2} V_{3}^{2}+\epsilon^{2} \alpha_{2}\left(\nabla^{2} V_{1}\right)^{2}\right. \\
\left.+\pi\left(\dot{\phi}-V_{1}\right)+\pi_{1}\left(\dot{V}_{1}-\nabla^{2} \phi-V_{2}\right)+\pi_{2}\left(\dot{V}_{2}-V_{3}\right)\right\} \\
=\int d^{D} x\left\{\pi \dot{\phi}+\pi_{1} \dot{V}_{1}+\pi_{2} \dot{V}_{2}-\left[\pi V_{1}+\pi_{1}\left(\nabla^{2} \phi+V_{2}\right)+\pi_{2} V_{3}\right.\right. \\
\left.\left.-\frac{1}{2} V_{1}^{2}+\frac{1}{2}(\nabla \phi)^{2}+U-\epsilon \alpha_{1} V_{2}^{2}+\epsilon^{2} \alpha_{2}\left[V_{3}^{2}-\left(\nabla V_{2}\right)^{2}\right]\right]\right\} .
\end{gathered}
$$

The procedure here mirrors the one for the first order case. We provide all the steps in order to illustrate the method better. The equations resulting from the action principle with respect to all the independent fields are

$$
\begin{array}{lll}
\frac{\delta \mathcal{S}}{\delta V_{3}} \approx 0 & \Rightarrow & \pi_{2} \approx-2 \epsilon^{2} \alpha_{2} V_{3}, \\
\frac{\delta \mathcal{S}}{\delta V_{2}} \approx 0 & \Rightarrow & \dot{\pi}_{2} \approx-\pi_{1}+2 \epsilon \alpha_{1} V_{2}-2 \epsilon^{2} \alpha_{2} \nabla^{2} V_{2}, \\
\frac{\delta \mathcal{S}}{\delta V_{1}} \approx 0 & \Rightarrow & \dot{\pi}_{1} \approx-\pi V_{1}, \\
\frac{\delta \mathcal{S}}{\delta \phi} \approx 0 & \Rightarrow \quad & \dot{\pi} \approx-\nabla^{2} \pi_{1}+\nabla^{2} \phi-U^{\prime}, \\
\frac{\delta \mathcal{S}}{\delta \pi} \approx 0 & \Rightarrow \quad \dot{\phi} \approx V_{1}, \\
\frac{\delta \mathcal{S}}{\delta \pi_{1}} \approx 0 \quad & \Rightarrow \quad \dot{V}_{1} \approx \nabla^{2} \phi+V_{2}, \\
\frac{\delta \mathcal{S}}{\delta \pi_{2}} \approx 0 & \Rightarrow & \dot{V}_{2} \approx V_{3} .
\end{array}
$$

Multiplying these equations by $\epsilon^{2}$ gives the leading order equations,

$$
\begin{aligned}
\epsilon^{2} \pi_{2} & \approx 0, \\
\epsilon^{2} \dot{\pi}_{2} & \approx-\epsilon^{2} \pi_{1}, \\
\epsilon^{2} \dot{\pi}_{1} & \approx \epsilon^{2}\left(-\pi+V_{1}\right), \\
\epsilon^{2} \dot{\pi} & \approx \epsilon^{2}\left(-\nabla^{2} \pi_{1}+\nabla^{2} \phi-U^{\prime}\right), \\
\epsilon^{2} \dot{\phi} & \approx \epsilon^{2} V_{1}, \\
\epsilon^{2} \dot{V}_{1} & \approx \epsilon^{2}\left(\nabla^{2} \phi+V_{2}\right), \\
\epsilon^{2} \dot{V}_{2} & \approx \epsilon^{2} V_{3} .
\end{aligned}
$$


The first of these is a constraint equation. Requiring its time derivative to vanish on-shell produces another constraint,

$$
\epsilon^{2} \dot{\pi}_{2} \approx-\epsilon^{2} \pi_{1} \approx 0
$$

Consistency of this constraint produces the next one,

$$
\epsilon^{2} \dot{\pi}_{2} \approx \epsilon^{2}\left(-\pi+V_{1}\right) \approx 0 \quad \Rightarrow \quad \epsilon^{2} V_{1} \approx \epsilon^{2} \pi
$$

Again requiring consistency produces a constraint,

$$
\epsilon^{2} \frac{d}{d t}\left(-\pi+V_{1}\right) \approx \epsilon^{2}\left(V_{2}+U^{\prime}\right) \approx 0 \quad \Rightarrow \quad \epsilon^{2} V_{2} \approx-\epsilon^{2} U^{\prime} .
$$

Finally, the consistency of this constraint determines the Lagrange multiplier,

$$
\epsilon^{2} \frac{d}{d t}\left(V_{2}+U^{\prime}\right) \approx \epsilon^{2}\left(V_{3}+U^{\prime \prime} \pi\right) \approx 0 \quad \Rightarrow \quad \epsilon^{2} V_{3} \approx-\epsilon^{2} U^{\prime \prime} \pi .
$$

After solving the leading order constraints we proceed to the fist order ones. Multiplying equations (3.66)-(3.72) by $\epsilon$, and making use of the leading order constraints gives the equations at first order

$$
\begin{aligned}
\epsilon \pi_{2} & \approx 0, \\
\epsilon \dot{\pi}_{2} & \approx-\epsilon \pi_{1}-2 \epsilon^{2} \alpha_{1} U^{\prime}, \\
\epsilon \dot{\pi}_{1} & \approx \epsilon\left(-\pi+V_{1}\right), \\
\epsilon \dot{\pi} & \approx \epsilon\left(-\nabla^{2} \pi_{1}+\nabla^{2} \phi-U^{\prime}\right), \\
\epsilon \dot{\phi} & \approx \epsilon V_{1}, \\
\epsilon \dot{V}_{1} & \approx \epsilon\left(\nabla^{2} \phi+V_{2}\right), \\
\epsilon \dot{V}_{2} & \approx \epsilon V_{3} .
\end{aligned}
$$

The first of these equations is a constraint. Its consistency generates another constraint,

$$
\epsilon \dot{\pi}_{2} \approx-\epsilon \pi_{1}-2 \epsilon^{2} \alpha_{1} U^{\prime} \approx 0 \quad \Rightarrow \quad \epsilon \pi_{1} \approx-2 \epsilon^{2} U^{\prime}
$$

Its consistency generates the next constraint,

$$
\epsilon \frac{d}{d t}\left(\pi_{1}+2 \epsilon \alpha_{1} U^{\prime}\right) \approx 0 \quad \Rightarrow \quad \epsilon V_{1} \approx \epsilon \pi-2 \epsilon^{2} \alpha_{1} U^{\prime \prime} \pi
$$

which by consistency requirements generates yet another one

$$
\epsilon \frac{d}{d t}\left(V_{1}-\pi+2 \epsilon \alpha_{1} U^{\prime \prime} \pi\right) \approx 0 \quad \Rightarrow \quad \epsilon V_{2} \approx-\epsilon U^{\prime}+2 \epsilon^{2} \alpha_{1}\left(U^{\prime \prime \prime}\left[-\pi^{2}+(\nabla \phi)^{2}\right]+U^{\prime \prime} U^{\prime}\right) .
$$

Finally consistency of this constraint determines $V_{3}$ at first order, but we do not require this to construct the reduced action. 
In the last stage of solving the constraints we turn to the second order equations (3.66)(3.72). By successively requiring consistency of constraints solve the perturbative constraints fully. Here we give just the terms that do not cancel when plugged into the action,

$$
\begin{aligned}
& \pi_{2} \approx 2 \epsilon^{2} \alpha_{2} U^{\prime \prime} \pi \\
& \pi_{1} \approx-2 \epsilon \alpha_{1} U^{\prime}+2 \epsilon^{2}\left(\alpha_{2}+2 \alpha_{1}^{2}\right)\left(U^{\prime \prime \prime}\left[-\pi^{2}+(\nabla \phi)^{2}\right]+U^{\prime \prime} U^{\prime}\right), \\
& V_{1} \approx \pi-2 \epsilon \alpha_{1} U^{\prime \prime} \pi+\mathcal{O}\left(\epsilon^{2}\right), \\
& V_{2} \approx-U^{\prime}+\mathcal{O}(\epsilon) \\
& V_{3} \approx-U^{\prime \prime} \pi+\mathcal{O}(\epsilon) .
\end{aligned}
$$

These are now plugged into the extended action (3.65) as strong equalities, resulting in the reduced phase space action, which after some partial integrations can be written as

$$
\begin{aligned}
\mathscr{S}_{\text {red }}= & \int d^{D} x\left\{\left[\pi+2 \epsilon \alpha_{1} U^{\prime \prime} \pi+2 \epsilon^{2}\left(\alpha_{2}+2 \alpha_{1}^{2}\right)\left(\frac{1}{3} U^{(4)} \pi^{3}-U^{(4)}(\nabla \phi)^{2} \pi\right.\right.\right. \\
& \left.\left.+2 \nabla\left(U^{\prime \prime \prime} \pi \nabla \phi\right)-\left[U^{\prime \prime \prime} U^{\prime}+2\left(U^{\prime \prime}\right)^{2}\right] \pi\right)\right] \dot{\phi} \\
& -\left[\frac{1}{2} \pi^{2}+\frac{1}{2}(\nabla \phi)^{2}+U+\epsilon \alpha_{1}\left(2 U^{\prime \prime}(\nabla \phi)^{2}+\left(U^{\prime}\right)^{2}\right)-\alpha_{2} \epsilon^{2}\left(U^{\prime \prime}\right)^{2}(\nabla \phi)^{2}\right. \\
& \left.\left.+2 \epsilon^{2}\left(\alpha_{2}+2 \alpha_{1}^{2}\right)\left(U^{\prime \prime \prime}\left[-\pi^{2}+(\nabla \phi)^{2}\right]+U^{\prime \prime} U^{\prime}\right)\left(\nabla^{2} \phi-U^{\prime}\right)-\left(\alpha_{2}+2 \alpha_{1}^{2}\right) \epsilon^{2}\left(U^{\prime \prime}\right)^{2} \pi^{2}\right]\right\} .
\end{aligned}
$$

The reduced phase space action at second order comes with the noncanonical symplectic part, but such that it can be canonicalized by redefining the conjugate momentum field, just as we had at the first order reduction. We do not bother with canonicalizing the phase space action, but proceed to construct the reduced configuration space action. First we solve for the conjugate momentum field

$$
\frac{\delta \mathscr{S}_{\text {red }}}{\delta \pi} \approx 0 \quad \Rightarrow \quad \pi \approx \dot{\phi}+2 \epsilon \alpha_{1} U^{\prime \prime} \dot{\phi}+\mathcal{O}\left(\epsilon^{2}\right),
$$

and then plug it into the phase space action (3.99). Thus we obtain the reduced configuration space action, which after some number of partial integrations and groupings of the terms can be written in a manifestly Lorentz invariant form,

$$
\begin{aligned}
S_{\text {red }}= & \int d^{D} x\left\{-\frac{1}{2}\left[1+4 \epsilon \alpha_{1} U^{\prime \prime}-8\left(\alpha_{2}+2 \alpha_{1}^{2}\right) U^{\prime \prime \prime} U^{\prime}-2\left(3 \alpha_{2}+4 \alpha_{1}^{2}\right)\left(U^{\prime \prime}\right)^{2}\right]\left(\partial_{\mu} \phi\right)\left(\partial^{\mu} \phi\right)\right. \\
& \left.-U-\epsilon \alpha_{1}\left(U^{\prime}\right)^{2}+2\left(\alpha_{2}+2 \alpha_{1}^{2}\right) U^{\prime \prime}\left(U^{\prime}\right)^{2}-2\left(\alpha_{2}+2 \alpha_{1}^{2}\right) U^{\prime \prime \prime}\left(\partial_{\mu} \phi\right)\left(\partial^{\mu} \phi\right)(\square \phi)\right\} .
\end{aligned}
$$

Apart from renormalizing the kinetic term, and modifying the potential already encountered at first order, the effect of reduction the considered higher derivative is also to introduce a new term - the last one in the action above. This term technically is a higher derivative one due to the presence of d'Alembertian operator, but it is not a genuine higher derivative terms, since the higher time derivative in it can be removed by partial integration, and are only introduced for the sake of manifest Lorentz invariance. Moreover, the 
very method used to derive it guarantees no higher derivatives in the final result. It is worth noting that derivative reduction introduces new types of terms containing four derivatives into the reduced action, but the kind that are not genuine higher derivative ones.

\subsubsection{Derivative reduction via field redefinition}

With a bit of work one can see that the field transformation,

$$
\phi \rightarrow \phi-\epsilon \alpha_{1} \square \phi+\frac{3}{2} \epsilon^{2} \alpha_{1}^{2} \square \square \phi+2 \epsilon^{2} \alpha_{1}^{2} U^{\prime \prime} \square \phi+2 \epsilon^{2} \alpha_{2} \square\left[\square \phi+U^{\prime}\right],
$$

removes all the genuine higher derivative terms from the configuration space action (3.61), and transforms it into

$$
\begin{gathered}
S \rightarrow \int d^{D} x\left\{-\frac{1}{2}\left[1+2 \epsilon \alpha_{1} U^{\prime \prime}-2 \epsilon^{2}\left(\alpha_{2}+2 \alpha_{1}^{2}\right)\left(U^{\prime \prime}\right)^{-} 4 \epsilon^{2} \alpha_{1}^{2} U^{\prime \prime \prime} U^{\prime}\right]\left(\partial_{\mu} \phi\right)\left(\partial^{\mu} \phi\right)\right. \\
\left.-U-\frac{3}{2} \epsilon^{2} \alpha_{1}^{2} U^{\prime \prime \prime} U^{\prime}\left(\partial_{\mu} \phi\right)\left(\partial^{\mu} \phi\right)(\square \phi)\right\} .
\end{gathered}
$$

This action is not in the form of the reduced one (3.101) obtained via perturbative constraints method. But again, this is not an inconsistency, since an additional field redefinition

$\phi \rightarrow \phi+\epsilon \alpha_{1} U^{\prime}-\frac{5}{2} \epsilon^{2} \alpha_{1}^{2} U^{\prime \prime \prime}\left(\partial_{\mu} \phi\right)\left(\partial^{\mu} \phi\right)-\frac{9}{2} \epsilon^{2} \alpha_{1}^{2} U^{\prime \prime} U^{\prime}-2 \epsilon^{2} \alpha_{2} U^{\prime \prime \prime}\left(\partial_{\mu} \phi\right)\left(\partial^{\mu} \phi\right)-2 \epsilon^{2} \alpha_{2} U^{\prime \prime} U^{\prime}$,

bring the above action precisely to the form of (3.101). The field redefinition method is consistent with the perturbative constraints method.

\subsection{Derivative reduction at third order - Part I}

In order to illustrate the points made at this section we consider the leading order action (3.2) with the only nonvanishing higher order correction (3.27) coming in at the third order EFT expansion,

$$
S=\int d^{D} x\left\{-\frac{1}{2}\left(\partial_{\mu} \phi\right)\left(\partial^{\mu} \phi\right)-U+\epsilon^{3} \alpha_{3}(\square \square \phi)(\square \square \phi)\right\} .
$$

At this order we make the point that field redefinitions are necessary in order to define an equivalent lower derivative, manifestly Lorentz invariant, configuration space action formulation of the higher derivative system.

\subsubsection{Reduction at the equation of motion level}

The equation of motion descending from the action (3.105) is

$$
\square \phi-U^{\prime}+2 \epsilon^{3} \alpha_{3} \square \square \square \square \phi=0 .
$$

Just as in the cases of the first and the second order reduction, we make use of the lower order equation to reduce the derivative order of the higher order part of the equation. The 
resulting equation takes the form

$$
\begin{aligned}
\square \phi-U^{\prime} & +2 \epsilon^{3} \alpha_{3}\left\{A(\phi)+B(\phi) \times\left(\partial_{\mu} \phi\right)\left(\partial^{\mu} \phi\right)+C(\phi) \times\left(\partial_{\mu} \phi\right)\left(\partial^{\mu} \phi\right)\left(\partial_{\nu} \phi\right)\left(\partial^{\nu} \phi\right)\right. \\
& +D(\phi) \times\left(\partial_{\mu} \partial_{\nu} \phi\right)\left(\partial^{\mu} \phi\right)\left(\partial^{\nu} \phi\right)+E(\phi) \times\left(\partial_{\mu} \partial_{\nu} \phi\right)\left(\partial^{\mu} \partial^{\nu} \phi\right) \\
& +8 U^{(5)}\left(\partial_{\mu} \partial_{\nu} \partial_{\rho} \phi\right)\left(\partial^{\mu} \phi\right)\left(\partial^{\nu} \phi\right)\left(\partial^{\rho} \phi\right)+24 U^{(4)}\left(\partial_{\mu} \partial_{\nu} \partial_{\rho} \phi\right)\left(\partial^{\mu} \partial^{\nu} \phi\right)\left(\partial^{\rho} \phi\right) \\
& \left.+4 U^{\prime \prime \prime}\left(\partial_{\mu} \partial_{\nu} \partial_{\rho} \phi\right)\left(\partial^{\mu} \partial^{\nu} \partial^{\rho} \phi\right)\right\}=0 .
\end{aligned}
$$

The functions $A-E$ are composed out of various derivatives of the potential function $U$, and are not important for this discussion. The important thing to notice is that the last three terms in the brackets are of third order in derivatives, and that there is no way of reducing their (time) derivative order while maintaining Lorentz covariance. In the first and second order derivative reductions whenever we needed to use the lower order equation in the higher order part we were always encountering second time derivatives in the combination with the Laplacian so that they form the d'Alembertian $\square$. Here this is no longer the case, and if we want to reduce third derivatives above we need to pick out second time derivatives individually, which will break manifest covariance. This example teaches us that reducing the derivative order at the level of the equations of motion is not always compatible with the symmetries of the system.

Note also that a field redefinition including higher derivatives,

$$
\phi \rightarrow \phi-4 \epsilon^{3} \alpha_{3} U^{\prime \prime \prime}\left[\left(\partial_{\mu} \partial_{\nu} \phi\right)\left(\partial^{\mu} \partial^{\nu} \phi\right)+2 U^{(4)}\left(\partial_{\mu} \partial_{\nu} \phi\right)\left(\partial^{\mu} \phi\right)\left(\partial^{\nu} \phi\right)\right],
$$

precisely removes the third derivatives from the equation of motion (and modifies functions $A-E$ ), while preserving covariance. There seems to be no way around making field redefinitions when perturbatively reducing the derivative order while maintaining covariance.

\subsubsection{Reduction via perturbative constraints}

Similar maladies afflicting derivative reduction at the level of the equation of motion exhibited in the previous subsection affect the method of perturbative constraints. It will be shown here that field redefinitions are unavoidable.

The starting point is again the extended action, with time derivatives promoted to independent fields,

$$
\dot{\phi} \rightarrow V_{1}, \quad-\square \phi=\dot{V}_{1}-\nabla^{2} \phi \rightarrow V_{2}, \quad-\partial_{0} \square \phi=\dot{V}_{2} \rightarrow V_{3}, \quad \square \square \phi=\dot{V}_{3}-\nabla^{2} V_{2} \rightarrow V_{4},
$$

and Lagrange multipliers $\pi, \pi_{1}, \pi_{2}$, and $\pi_{3}$ introduced to ensure on-shell equivalence,

$$
\begin{aligned}
& \mathcal{S}=\int d^{D} x\left\{\frac{1}{2} V_{1}^{2}-\right. \frac{1}{2}(\nabla \phi)^{2}-U+\epsilon^{3} \alpha_{3} V_{4}^{2}+\pi\left(\dot{\phi}-V_{1}\right)+\pi_{1}\left(\dot{V}_{1}-\nabla^{2} \phi-V_{2}\right) \\
&\left.+\pi_{2}\left(\dot{V}_{2}-V_{3}\right)+\pi_{3}\left(\dot{V}_{3}-\nabla^{2} V_{2}-V_{4}\right)\right\} \\
&=\int d^{D} x\left\{\pi \dot{\phi}+\pi_{1} \dot{V}_{1}+\pi_{2} \dot{V}_{2}+\pi_{3} \dot{V}_{3}-\left[\pi V_{1}+\pi_{2}\left(\nabla^{2} \phi+V_{2}\right)+\pi_{2} V_{3}\right.\right. \\
&\left.\left.+\pi_{3}\left(\nabla^{2} V_{2}+V_{4}\right)-\frac{1}{2} V_{1}^{2}+\frac{1}{2}(\nabla \phi)^{2}+U-\epsilon^{3} \alpha_{3} V_{4}^{2}\right]\right\} .
\end{aligned}
$$


We do not bother here with presenting step-by-step how the perturbative constraints are imposed order by order, this should be clear from examples of the first and second order reductions, and the expressions at third order are rather lengthy while bringing noting new to the discussion. Instead we just cite the solutions of the perturbative second-class constraints needed to reduce the derivative order, and obtain the lower derivative phase space action,

$$
\begin{aligned}
& \pi_{3} \approx-\epsilon^{3} \alpha_{3}\left(U^{\prime \prime}\left[\pi^{2}-(\nabla \phi)^{2}\right]-U^{\prime \prime} U^{\prime}\right), \\
& \pi_{2} \approx-2 \epsilon^{3} \alpha_{3}\left\{U^{(4)} \pi\left[-\pi^{2}+(\nabla \phi)^{2}\right]-2 U^{\prime \prime \prime} \pi\left[\nabla^{2} \phi-U^{\prime}\right]\right. \\
& \left.+2 U^{\prime \prime \prime}(\nabla \phi)(\nabla \pi)+\left[U^{\prime \prime} U^{\prime}\right]^{\prime} \pi\right\}, \\
& \pi_{1} \approx 2 \epsilon^{3} \alpha_{3}\left\{4 U^{\prime \prime \prime}(\nabla \pi)^{2}+8 U^{(4)} \pi(\nabla \pi)(\nabla \phi)-U^{(5)}\left[\pi^{2}-(\nabla \phi)^{2}\right]^{2}\right. \\
& -U^{(4)}\left[4 \pi^{2} \nabla^{2} \phi+U^{\prime}(\nabla \phi)^{2}+2(\nabla \phi) \nabla(\nabla \phi)^{2}\right] \\
& +U^{\prime \prime \prime}\left[-2\left(\nabla^{2} \phi-U^{\prime}\right)^{2}+2(\nabla \phi) \nabla\left[\nabla^{2} \phi-U^{\prime}\right]-\nabla^{2}(\nabla \phi)^{2}\right] \\
& \left.+\left[U^{\prime \prime} U^{\prime}\right]^{\prime \prime \prime}\left[\pi^{2}-(\nabla \phi)^{2}\right]+\left[5 U^{(4)} U^{\prime}+2 U^{\prime \prime \prime} U^{\prime \prime}\right] \pi^{2}-\left[U^{\prime \prime} U^{\prime}\right]^{\prime} U^{\prime}\right\}, \\
& V_{4} \approx-U^{\prime \prime \prime}\left[\pi^{2}-(\nabla \phi)^{2}\right]-U^{\prime \prime} U^{\prime}+\mathcal{O}(\epsilon) \\
& V_{3} \approx-\epsilon^{3} U^{\prime}, \\
& V_{2} \approx-U^{\prime}+\mathcal{O}(\epsilon) \text {, } \\
& V_{1} \approx \pi+\mathcal{O}(\epsilon) \text {. }
\end{aligned}
$$

Promoting these to strong equalities and plugging them in the extended action (3.110) gives the phase space reduced action, which takes the form

$$
\mathscr{S}_{\text {red }}=\int d^{D} x\left\{8 \epsilon^{3} \alpha_{3}\left[U^{\prime \prime \prime}(\nabla \pi)^{2}+2 U^{(4)} \pi(\nabla \pi)(\nabla \phi)\right] \dot{\pi}+\left(\pi+\epsilon^{3} \alpha_{3} \Pi[\phi, \pi]\right) \dot{\phi}-H[\phi, \pi]\right\},
$$

where $\Pi$ is a function of $\phi, \pi$, and their spatial derivatives, and so is the Hamiltonian $H$. The method of derivative reduction via perturbative second-class constraints accomplishes exactly what it is designed to do - produces a phase space formulation of a lower derivative system capturing completely the perturbative solutions of the original system to order $\mathcal{O}\left(\epsilon^{4}\right)$. But phase space actions are considerably less convenient to work with than the configuration space ones, and it is desirable to construct one here.

The specific form of the phase space action (3.118) is of interest for the discussion here, but rather just the symplectic part. The fact that the symplectic part appears in a non-canonical form is by now not surprising, since we have encountered similar situations at lower orders in derivative reduction. The novel feature here is that the symplectic part cannot be canonicalized by only redefining the conjugate momentum field. The part containing the function $\Pi$ can easily be removed by

$$
\pi \rightarrow \pi-\epsilon^{3} \alpha_{3} \Pi
$$

but the part of (3.118) containing $\dot{\pi}$, cannot be removed this way. There is no way to rewrite it so that the time derivative is only on the scalar field $\phi$. This is the behaviour 
mentioned in section 2 that appears in systems with multiple degrees of freedom. A field has infinitely many local degrees of freedom, and their coupling is expressed through gradients. It is precisely gradients acting on the conjugate momentum field that are preventing the canonicalization of the action.

If we insist on not redefining the scalar field itself, this also means there is no configuration space formulation of the action (3.118) solely in terms of the scalar field $\phi$. There perhaps is a Lagrangian description with additional constraints, but they are likely to require explicit perturbative treatment, which defeats the purpose of this construction.

The action (3.118) can, of course, be canonicalized by the scalar field redefinition,

$$
\phi \rightarrow \phi+8 \epsilon^{3} \alpha_{3}\left[U^{\prime \prime \prime}(\nabla \phi)^{2}+2 U^{(4)} \pi(\nabla \pi)(\nabla \phi)\right] .
$$

After performing this, we will surely be able to find a configuration space action. But as soon as we start making such fields redefinitions in the phase space action, we do not really know (unless we investigate it in detail) whether we are preserving Lorentz covariance or not, and things become rather complicated.

The conclusion here is that field redefinitions are unavoidable when looking for a lower derivative configuration space action formulation of the systems where higher derivatives enter perturbatively. This being the case, we might as well consider field redefinitions of the configuration space action (3.105) right from the beginning.

\subsubsection{Reduction via field redefinition}

The field redefinition method when employed at the first and second order yielded the equivalent results as reducing derivative order in the equation of motion, and as the perturbative constraints method. Moreover, it was somewhat easier and faster to implement. At the third order the difference in the practicality of the methods becomes huge. In any case we need to employ field redefinitions, but doing it directly on the configuration space action is now tremendously faster and more straightforward. With comparatively much less work one can see that the scalar field redefinition

$$
\phi \rightarrow \phi-\epsilon^{3} \alpha^{3} \square \square\left[\square \phi-U^{\prime}\right]-\epsilon^{3} \alpha_{3}\left(U^{\prime \prime}\right)^{2}(\square \phi),
$$

transforms the action (3.105) into

$$
\begin{aligned}
S \rightarrow \int d^{D} x\{- & \frac{1}{2}\left(1+2 \epsilon^{3} \alpha_{3}\left[\left(U^{\prime \prime}\right)^{2} U^{\prime}\right]^{\prime}\right)\left(\partial_{\mu} \phi\right)\left(\partial^{\mu} \phi\right)-U \\
& \left.+\epsilon^{3} \alpha_{3}\left(U^{\prime \prime}\right)^{2}\left(\partial_{\mu} \phi\right)\left(\partial^{\mu} \phi\right)\left(\partial_{\nu} \phi\right)\left(\partial^{\nu} \phi\right)+2 \epsilon^{3} \alpha_{3} U^{\prime \prime \prime} U^{\prime \prime}\left(\partial_{\mu} \phi\right)\left(\partial^{\mu} \phi\right)(\square \phi)\right\},
\end{aligned}
$$

removing all the genuine higher derivative terms, leaving only terms propagating three degrees of freedom.

\subsection{Derivative reduction at third order - Part II}

As a last example of this section we examine the leading order action (3.2) with a single higher order correction (3.21) from the third order EFT expansion,

$$
S=\int d^{D} x\left\{-\frac{1}{2}\left(\partial_{\mu} \phi\right)\left(\partial^{\mu} \phi\right)-U+\epsilon^{3} \alpha_{3}\left(\partial_{\mu} \phi\right)\left(\partial^{\mu} \phi\right)\left(\partial_{\nu} \partial_{\rho} \phi\right)\left(\partial^{\nu} \partial^{\rho} \phi\right)\right\} .
$$


The conclusions of the preceding subsection were that field redefinitions are generally inevitable in derivative reduction methods, therefore we consider only field redefinition here.

The higher order correction term in (3.123) is indeed a genuine higher derivative term. It is not difficult to recognize that no Lorentz covariant field redefinition will be able to remove it (though non-covariant redefinitions would allow for this [36]). At this point we draw the attention to the difference between genuine and non-genuine higher derivative terms, former carrying extra degrees of freedom, compared to the latter that do not but still contain higher (spatial) derivatives. One such non-genuine higher derivative term is

$$
\int d^{D} x\left(\partial_{\mu} \phi\right)\left(\partial^{\nu} \phi\right)\left[(\square \phi)(\square \phi)-\left(\partial_{\mu} \partial_{\nu} \phi\right)\left(\partial^{\mu} \partial^{\nu} \phi\right)\right],
$$

which is a specific flat space specialization of the fourth Horndeski action [8]. We can rewrite the action (3.123) in terms of it,

$$
\begin{aligned}
S=\int d^{D} x\{- & \frac{1}{2}\left(\partial_{\mu} \phi\right)\left(\partial^{\mu} \phi\right)-U-\epsilon^{3} \alpha_{3}\left(\partial_{\mu} \phi\right)\left(\partial^{\nu} \phi\right)\left[(\square \phi)(\square \phi)-\left(\partial_{\mu} \partial_{\nu} \phi\right)\left(\partial^{\mu} \partial^{\nu} \phi\right)\right] \\
& \left.+\epsilon^{3} \alpha_{3}\left(\partial_{\mu} \phi\right)\left(\partial^{\nu} \phi\right)(\square \phi)(\square \phi)\right\} .
\end{aligned}
$$

Now the only genuine higher derivative term in the action above can be taken the term in the bottom row, and that one is easily removed by the following field redefinition,

$$
\phi \rightarrow \phi-\epsilon^{3} \alpha_{3}\left(\partial_{\mu} \phi\right)\left(\partial^{\mu} \phi\right)(\square \phi),
$$

transforming the action to

$$
\begin{aligned}
S \rightarrow \int d^{D} x\{- & \frac{1}{2}\left(\partial_{\mu} \phi\right)\left(\partial^{\mu} \phi\right)-U-\epsilon^{3} \alpha_{3}\left(\partial_{\mu} \phi\right)\left(\partial^{\nu} \phi\right)\left[(\square \phi)(\square \phi)-\left(\partial_{\mu} \partial_{\nu} \phi\right)\left(\partial^{\mu} \partial^{\nu} \phi\right)\right] \\
& \left.+\epsilon^{3} \alpha_{3} U^{\prime}\left(\partial_{\mu} \phi\right)\left(\partial^{\nu} \phi\right)(\square \phi)\right\}
\end{aligned}
$$

where now the term in the bottom row is not genuinely higher derivative, and the whole action propagates no extra degrees of freedom. This field transformation teaches us that sometimes seemingly we do not need to eliminate a term by field transformation, but produce it in order to eliminate the genuine higher derivative behavior.

\subsection{Summary of the single scalar case study}

Studying different examples of perturbative derivative reductions in single scalar field systems we have learned several lessons summarized by the following points:

- Reducing derivative order at the level of equations of motion is ambiguous. Many different equations capture perturbatively expandable solutions to a given order, and only specific ones have an action formulation. Those are the ones that should be used when attempting to solve the truncated lower order equation exactly. 
- It is not always possible to reduce the derivative order at the equations of motion level completely, and at the same time retain Lorentz covariance. Field redefinitions are needed to complete the task in general. This point was proved in [36] for the case of multiple interacting scalar fields, and second derivatives in the action.

- Derivative reduction by perturbative second-class constraints always accomplishes what it is meant to do - remove spurious degrees of freedom and deliver a lower derivative (or non-genuine higher derivative) phase space action. In general there is no equivalent configuration space action corresponding to this phase space reduced one, unless we allow for additional non-covariant field redefinitions.

- Field redefinition method successfully eliminates genuine higher derivative terms from the configuration space action to a desired order, and is consistent with the first two methods.

- Genuine higher derivative corrections are mapped to lower order or non-genuine higher derivative corrections. These correctly capture the EFT corrections. Still, we need to know which higher derivative terms are not genuine, the reduction method does not tell us this.

- Removal of genuine higher derivative EFT corrections by field redefinition is generally not unique (but then again neither is any action where field redefinitions are allowed).

In conclusion, the field redefinition method is the preferred method for reducing genuine higher order EFT corrections.

\section{Derivative reduction in scalar-tensor theory}

In this section we apply the lessons learned in the single scalar case study of the preceding section, and employ the field redefinition method to the higher order EFT corrections of a scalar-tensor theory. Field redefinitions have been investigated in the context of EFTs of perturbations around time-dependent backgrounds, and have been used to remove spurious higher derivative corrections (see e.g. [46-48]). Here we address a somewhat different issue of higher derivative corrections to the background itself. Scalar-tensor theories are particularly suited for our purposes, since many of the non-genuine higher derivative terms have been worked out and classified, first within Horndeski theory [8], and later beyond Horndeski $[12,13]$, and lately within the more general DHOST program [14-16]. This actually makes our job much easier, since we can concentrate only on removing the genuine higher derivative corrections.

Here we also work in $D$ spacetime dimensions, and use the following conventions: metric field is of positive signature, the Christoffel symbol is $\Gamma_{\mu}^{\alpha} \nu=\frac{1}{2} g^{\alpha \beta}\left(\partial_{\mu} g_{\nu \beta}+\partial_{\nu} g_{\mu \beta}-\right.$ $\left.\partial_{\beta} g_{\mu \nu}\right)$, the Riemann tensor $R^{\rho}{ }_{\mu \sigma \nu}=\partial_{\sigma} \Gamma_{\mu \nu}^{\rho}-\ldots$, the Ricci tensor $R_{\mu \nu}=R_{\mu \rho \nu}^{\rho}$, the Ricci scalar $R=R_{\mu}^{\mu}$, and $\nabla_{\mu}$ is the covariant derivative.

The leading order action in our scalar-tensor theory is assumed to be

$$
S^{(0)}=\int d^{D} x \sqrt{-g}\left\{\frac{1}{\kappa^{2}} f(\phi) R-\frac{1}{2} z(\phi) g^{\mu \nu}\left(\nabla_{\mu} \phi\right)\left(\nabla_{\nu} \phi\right)-u(\phi)\right\}, \quad \kappa=\sqrt{16 \pi G_{N}},
$$

and it propagates one scalar and two tensor degrees of freedom in $D=4$. 
The EFT expansion of corrections to this action is somewhat awkward with the metric dimensionless and the scalar field of mass dimension one. It is far more convenient to define a dimensionless scalar,

$$
\varphi=\kappa \phi,
$$

so that the leading order action reads

$$
S^{(0)}=\frac{1}{\kappa^{2}} \int d^{D} x \sqrt{-g}\left\{F(\varphi) R-\frac{1}{2} Z(\varphi) g^{\mu \nu}\left(\nabla_{\nu} \varphi\right)\left(\nabla_{\nu} \varphi\right)-\mathrm{U}(\varphi)\right\}
$$

with the identifications $F(\varphi)=f(\phi), Z(\varphi)=z(\phi), \mathrm{U}(\varphi)=u(\phi)$. Now the EFT expansion is naturally organized as an expansion in total number of derivatives. The leading order action contains terms with zero and two derivatives. The first order correction terms then contain four derivatives, and are one $\kappa^{2}$ order higher then the leading order action,

$$
S^{(1)}=\sum_{n=1}^{9} S_{n}^{(1)},
$$

where all the independent terms containing four derivatives are

$$
\begin{aligned}
S_{1}^{(1)} & =\int d^{D} x \sqrt{-g} f_{1}(\varphi)\left(\nabla_{\mu} \varphi\right)\left(\nabla^{\mu} \varphi\right)\left(\nabla_{\nu} \varphi\right)\left(\nabla^{\nu} \varphi\right), \\
S_{2}^{(1)} & =\int d^{D} x \sqrt{-g} f_{2}(\varphi)\left(\nabla_{\mu} \varphi\right)\left(\nabla^{\mu} \varphi\right)(\square \varphi), \\
S_{3}^{(1)} & =\int d^{D} x \sqrt{-g} f_{3}(\varphi)(\square \varphi)(\square \varphi), \\
S_{4}^{(1)} & =\int d^{D} x \sqrt{-g} f_{4}(\varphi)\left(\nabla_{\mu} \varphi\right)\left(\nabla^{\mu} \varphi\right) R, \\
S_{5}^{(1)} & =\int d^{D} x \sqrt{-g} f_{5}(\varphi)\left(\nabla_{\mu} \varphi\right)\left(\nabla_{\nu} \varphi\right) R^{\mu \nu}, \\
S_{6}^{(1)} & =\int d^{D} x \sqrt{-g} f_{6}(\varphi)(\square \varphi) R \\
S_{7}^{(1)} & =\int d^{D} x \sqrt{-g} f_{7}(\varphi) R^{2}, \\
S_{8}^{(1)} & =\int d^{D} x \sqrt{-g} f_{8}(\varphi) R_{\mu \nu} R^{\mu \nu}, \\
S_{9}^{(1)} & =\int d^{D} x \sqrt{-g} f_{9}(\varphi) R_{\mu \nu \rho \sigma} R^{\mu \nu \rho \sigma},
\end{aligned}
$$

and $f_{1}-f_{9}$ are arbitrary functions of the scalar field. All the other scalar-tensor actions containing four derivatives that one can write down can be expressed in terms if the nine above my making use of partial integrations, commutators of covariant derivatives, and the Bianchi identities. This example of a scalar-tensor theory with dimension four corrections was considered a while ago by Weinberg [43] where it was determined what is the maximal number of dimension 4 corrections that can be removed by field redefinitions. Here we investigate a somewhat different question of removing genuine higher derivative operators.

Not all of the terms (4.5)-(4.13) are genuinely higher derivative ones, in the sense that they do not introduce additional degrees of freedom compared to the three present 
in the leading order action. Luckily, the most general scalar-tensor action containing four derivatives and propagating only three degrees of freedom is known. Most of it is captured by the Horndeski action [8] specialized to four derivatives, ${ }^{7}$

$$
\begin{aligned}
& S_{2}^{\mathrm{H}}=\int d^{D} x \sqrt{-g} g_{2}(\varphi)\left(\nabla_{\mu} \varphi\right)\left(\nabla^{\mu} \varphi\right)\left(\nabla_{\nu} \varphi\right)\left(\nabla^{\nu} \varphi\right), \\
& S_{3}^{\mathrm{H}}=\int d^{D} x \sqrt{-g} g_{3}(\varphi)\left(\nabla_{\mu} \varphi\right)\left(\nabla^{\mu} \varphi\right)(\square \varphi), \\
& S_{4}^{\mathrm{H}}=\int d^{D} x \sqrt{-g} g_{4}(\varphi)\left\{\left(\nabla_{\mu} \varphi\right)\left(\nabla^{\mu} \varphi\right) R-2\left[(\square \varphi)(\square \varphi)-\left(\nabla_{\mu} \nabla_{\nu} \varphi\right)\left(\nabla^{\mu} \nabla^{\nu} \varphi\right)\right]\right\} .
\end{aligned}
$$

The last of these actions can be rearranged into a more convenient form

$$
\begin{aligned}
S_{4}^{\mathrm{H}}=\int d^{D} x \sqrt{-g}\left\{-2 g_{4}(\varphi)\right. & G^{\mu \nu}\left(\nabla_{\mu} \varphi\right)\left(\nabla_{\nu} \varphi\right)+3 g_{4}^{\prime}(\varphi)\left(\nabla_{\mu} \varphi\right)\left(\nabla^{\mu} \varphi\right)(\square \varphi) \\
+ & \left.g_{4}^{\prime \prime}(\varphi)\left(\nabla_{\mu} \varphi\right)\left(\nabla^{\mu} \varphi\right)\left(\nabla_{\nu} \varphi\right)\left(\nabla^{\nu} \varphi\right)\right\},
\end{aligned}
$$

so instead of it we will use a more convenient one

$$
\widetilde{S}_{4}^{\mathrm{H}}=\int d^{D} x \sqrt{-g} \widetilde{g}_{4}(\varphi) G^{\mu \nu}\left(\nabla_{\mu} \varphi\right)\left(\nabla_{\nu} \varphi\right),
$$

where $G_{\mu \nu}=R_{\mu \nu}-\frac{1}{2} g_{\mu \nu} R$ is the Einstein tensor. There is one more remaining action with four derivatives propagating only three degrees of freedom, and it is known from LanczosLovelock gravity [37, 38], and more generally from scalar-Gauss-Bonnet models [39],

$$
S^{\mathrm{SGB}}=\int d^{D} x \sqrt{-g} b(\varphi)\left[R^{2}-4 R_{\mu \nu} R^{\mu \nu}+R_{\mu \nu \rho \sigma} R^{\mu \nu \rho \sigma}\right]
$$

The actions (4.14), (4.15), (4.17), and (4.19) comprise the most general four-derivative action propagating one scalar and two tensor degrees of freedom.

Out of nine independent four-derivative actions (4.5)-(4.13) four do not need to be considered as higher derivative since we can instead write them as combinations forming actions (4.14), (4.15), (4.17), and (4.19) which are not genuinely higher derivative. They can be added to the leading order scalar-tensor action (4.3) as genuine EFT corrections not introducing spurious degrees of freedom. These considerations reduce the number of genuinely higher derivative terms we need to worry about to only five, which we relabel for convenience,

$$
\begin{aligned}
& S_{1}^{\mathrm{HD}}=S_{8}^{(1)}=\int d^{D} x \sqrt{-g} h_{1}(\varphi) R_{\mu \nu} R^{\mu \nu}, \\
& S_{2}^{\mathrm{HD}}=S_{7}^{(1)}=\int d^{D} x \sqrt{-g} h_{2}(\varphi) R^{2}, \\
& S_{3}^{\mathrm{HD}}=S_{6}^{(1)}=\int d^{D} x \sqrt{-g} h_{3}(\varphi)(\square \varphi) R, \\
& S_{4}^{\mathrm{HD}}=S_{5}^{(1)}=\int d^{D} x \sqrt{-g} h_{4}(\varphi)\left(\nabla_{\mu} \varphi\right)\left(\nabla^{\mu} \varphi\right) R, \\
& S_{5}^{\mathrm{HD}}=S_{3}^{(1)}=\int d^{D} x \sqrt{-g} h_{5}(\varphi)(\square \varphi)(\square \varphi) .
\end{aligned}
$$

${ }^{7}$ In the notation standard in the Horndeski theory literature we have (using conventions $X=\left(\partial_{\mu} \varphi\right)\left(\partial^{\mu} \varphi\right)$ )

$$
G_{2}(\varphi, X)=g_{2}(\varphi) X^{2}, \quad G_{3}(\varphi, X)=g_{3}(\varphi) X, \quad G_{4}(\varphi, X)=g_{4}(\varphi) X, \quad G_{5}(\varphi, X)=0 .
$$


We proceed to eliminate the five higher derivative corrections (4.20)-(4.24) to the action by field redefinitions of the dimensionless scalar and the metric,

$$
\varphi \rightarrow \varphi+\kappa^{2} \delta \varphi, \quad g_{\mu \nu} \rightarrow g_{\mu \nu}+\kappa^{2} \delta g_{\mu \nu} .
$$

The leading order action (4.3) transforms under these field redefinitions as

$$
S^{(0)} \rightarrow S^{(0)}+\kappa^{2}\left[\delta_{\varphi} S^{(0)}\right]+\kappa^{2}\left[\delta_{g} S^{(0)}\right],
$$

where the first subleading term on the right hand side descends form the scalar field redefinition,

$$
\kappa^{2}\left[\delta_{\varphi} S^{(0)}\right]=\int d^{D} x \sqrt{-g}\left\{F^{\prime} R+Z(\square \varphi)-\frac{1}{2} Z^{\prime}\left(\nabla_{\mu} \varphi\right)\left(\nabla^{\mu} \varphi\right)-U^{\prime}\right\} \delta \varphi,
$$

and the second one descends from the metric field redefinition,

$$
\begin{aligned}
\kappa^{2}\left[\delta_{g} S^{(0)}\right]= & \int d^{D} x \sqrt{-g}\left\{-F G_{\mu \nu}+F^{\prime}\left[\left(\nabla_{\mu} \nabla_{\nu} \varphi\right)-(\square \varphi) g_{\mu \nu}\right]\right. \\
& \left.+\frac{1}{2}\left[Z+2 F^{\prime \prime}\right]\left(\nabla_{\mu} \varphi\right)\left(\nabla_{\nu} \varphi\right)-\frac{1}{4}\left[Z+4 F^{\prime \prime}\right]\left(\nabla_{\rho} \varphi\right)\left(\nabla^{\rho} \varphi\right) g_{\mu \nu}-\frac{1}{2} U g_{\mu \nu}\right\} \delta g^{\mu \nu} .
\end{aligned}
$$

In order to accomplish the task of removing higher derivatives from the first order corrections (4.20)-(4.24) while preserving general covariance, quantities $\delta \varphi$ and $\delta g_{\mu \nu}$ have to contain two derivatives and have to be covariant themselves. Their general form is thus

$$
\begin{aligned}
\delta \varphi=C_{1} & \times(\square \varphi)+C_{2} \times\left(\nabla_{\mu} \varphi\right)\left(\nabla^{\mu} \varphi\right)+C_{3} \times R \\
\delta g_{\mu \nu}=C_{4} & \times g_{\mu \nu} R+C_{5} \times R_{\mu \nu}+C_{6} \times g_{\mu \nu}(\square \varphi) \\
& +C_{7} \times\left(\nabla_{\mu} \nabla_{\nu} \varphi\right)+C_{8} \times g_{\mu \nu}\left(\nabla_{\rho} \varphi\right)\left(\nabla^{\rho} \varphi\right)+C_{9} \times\left(\nabla_{\mu} \varphi\right)\left(\nabla_{\nu} \varphi\right),
\end{aligned}
$$

where the coefficients $C_{1}-C_{9}$ are functions of the scalar field, $C_{i}=C_{i}(\varphi)$. We plug these into the expressions for the transformation of leading order action, (4.27) and (4.28), and after performing some partial integrations and making use of the Bianchi identity, the transformed leading order action is put into the form

$$
S^{(0)} \rightarrow S^{(0)}+\int d^{D} x \sqrt{-g}\left\{\sum_{A} B_{A}(\varphi) \times \mathcal{R}_{A}\left[g_{\mu \nu}, \nabla_{\mu} \varphi\right]\right\},
$$

where all the terms $\mathcal{R}_{A}$ dependent on the metric and the covariant derivatives of the scalar field, and the scalar dependent functions $B_{A}$ are given in table 1.

We need to choose the nine coefficient functions $C_{i}$ 's so as to absorb five distinct terms (4.20)-(4.24), and the way to do this is not unique. In particular, the first five rows of table 1 need to absorb the genuine higher derivative terms. For the argument that we intend to make here it suffices to demonstrate that one particular solution exists,

$$
\begin{aligned}
& C_{2}=C_{6}=C_{7}=C_{9}=0, \quad C_{1}=-\frac{h_{5}}{Z}, \quad C_{5}=\frac{h_{1}}{F}, \\
& C_{4}=\frac{-Z h_{1}+2 Z h_{2}-2 F^{\prime} h_{3}+\frac{2\left(F^{\prime}\right)^{2}}{Z} h_{5}}{(D-2) Z F+2(D-1)\left(F^{\prime}\right)^{2}}, \quad C_{3}=\frac{1}{Z}\left\{-h_{3}+\frac{F^{\prime}}{Z} h_{5}+(D-1) F^{\prime} C_{4}\right\}, \\
& C_{8}=\frac{-2}{(D-2) F}\left\{h_{4}+\frac{F^{\prime}}{F} h_{1}^{\prime}-\frac{\left(F^{\prime}\right)^{2}}{2 F^{2}} h_{1}-\frac{Z^{\prime}}{2} C_{3}-\frac{1}{4}\left[(D-2) Z+4(D-1) F^{\prime \prime}\right] C_{4}\right\} .
\end{aligned}
$$




\begin{tabular}{|c|c|}
\hline $\mathcal{R}_{A}$ & $B_{A}(\varphi)$ \\
\hline$R_{\mu \nu} R^{\mu \nu}$ & $-C_{5} F$ \\
\hline$R^{2}$ & $C_{3} F^{\prime}+\left(\frac{D-2}{2}\right) C_{4} F+\frac{1}{2} C_{5} F$ \\
\hline$R(\square \varphi)$ & $C_{1} F^{\prime}+C_{3} Z-(D-1) C_{4} F^{\prime}+\left(\frac{D-2}{2}\right) C_{6} F$ \\
\hline$R\left(\nabla_{\mu} \varphi\right)\left(\nabla^{\mu} \varphi\right)$ & $C_{2} F^{\prime}-\frac{1}{2} C_{3} Z^{\prime}-\frac{1}{4} C_{4}\left[(D-2) Z+4(D-1) F^{\prime \prime}\right]+\frac{1}{2} C_{5}^{\prime} F^{\prime}$ \\
& $-\frac{1}{2} C_{7} F^{\prime}+\left(\frac{D-2}{2}\right) C_{8} F$ \\
\hline$(\square \varphi)(\square \varphi)$ & $C_{1} Z-(D-1) C_{6} F^{\prime}$ \\
\hline$G^{\mu \nu}\left(\nabla_{\mu} \varphi\right)\left(\nabla_{\nu} \varphi\right)$ & $\frac{1}{2}\left[C_{5} Z-2 C_{5}^{\prime} F^{\prime}\right]+C_{7}^{\prime} F-C_{9} F$ \\
\hline$\left(\nabla_{\mu} \varphi\right)\left(\nabla^{\mu} \varphi\right)(\square \varphi)$ & $-\frac{1}{2} C_{1} Z^{\prime}+C_{2} Z-\frac{1}{4} C_{6}\left[(D-2) Z+4(D-1) F^{\prime \prime}\right]$ \\
& $+\frac{1}{2}\left[3 C_{7}^{\prime} F^{\prime}-C_{7} Z\right]-(D-1) C_{8} F^{\prime}-\frac{3}{2} C_{9} F^{\prime}$ \\
\hline$\left(\nabla_{\mu} \varphi\right)\left(\nabla^{\mu} \varphi\right)\left(\nabla_{\nu} \varphi\right)\left(\nabla^{\nu} \varphi\right)$ & $-\frac{1}{2} C_{2} Z^{\prime}+\frac{1}{4}\left\{2\left[C_{7}^{\prime} F^{\prime}\right]^{\prime}-\left[C_{7} Z\right]^{\prime}\right\}-\frac{1}{4}\left[(D-2) Z+4(D-1) F^{\prime \prime}\right]$ \\
& $+\frac{1}{4}\left\{C_{9} Z-2\left[C_{9} F^{\prime}\right]^{\prime}\right\}$ \\
\hline$R$ & $-C_{3} U^{\prime}-\frac{D}{2} C_{4} U-\frac{1}{2} C_{5} U$ \\
\hline$\left(\nabla_{\mu} \varphi\right)\left(\nabla^{\mu} \varphi\right)$ & {$\left[C_{1} U^{\prime}\right]^{\prime}-C_{2} U^{\prime}+\frac{D}{2}\left[C_{6} U\right]^{\prime}+\frac{1}{2}\left[C_{7} U\right]^{\prime}-\frac{D}{2} C_{8} U-\frac{1}{2} C_{9} U$} \\
\hline
\end{tabular}

Table 1. All the terms appearing in the transformation of the leading order action (4.31) due to field redefinitions (4.25), (4.29), (4.30).

Therefore, the first order correction in the EFT expansion of the scalar-tensor theory is captured correctly by the most general scalar-tensor theory with at most four derivatives that contains only three (in $D=4$ ) degrees of freedom,

$$
\begin{aligned}
S= & \int d^{D} x \sqrt{-g}\left\{\frac{1}{\kappa^{2}}\left[F_{1} R-\frac{1}{2} F_{2}\left(\nabla_{\mu} \varphi\right)\left(\nabla^{\mu} \varphi\right)-F_{3}\right]+F_{4}\left(\nabla_{\mu} \varphi\right)\left(\nabla^{\mu} \varphi\right)\left(\nabla_{\nu} \varphi\right)\left(\nabla^{\nu} \varphi\right)\right. \\
& \left.+F_{5}\left(\nabla_{\mu} \varphi\right)\left(\nabla^{\mu} \varphi\right)(\square \varphi)+F_{6} G^{\mu \nu}\left(\nabla_{\mu} \varphi\right)\left(\nabla_{\nu} \varphi\right)+F_{7}\left(R^{2}-4 R_{\mu \nu} R^{\mu \nu}+R_{\mu \nu \rho \sigma} R^{\mu \nu \rho \sigma}\right)\right\},
\end{aligned}
$$

where $F_{1}-F_{7}$ are some functions of the scalar field, $F_{i}=F_{i}(\varphi)$ that in principle have an expansion in $\kappa^{2}$. This action is a subset of Horndeski actions. In fact, it is possible to set $F_{4}$ and $F_{5}$ to zero by further field redefinitions as found in [43].

\section{Conclusions}

The aim of this paper was to examine the nature of higher derivative corrections in the effective field theory expansions. The point of view that we take on them is that they arise 
as a peculiarity of the approximation scheme of local derivative expansion employed in the EFT expansion. The higher derivative corrections cannot be treated on the same footing as the lower derivative leading order action describing the system. Rather, their effect should be taken into account in the same spirit in which they arise - perturbatively. That implies capturing their effects with terms that do not introduce extra degrees of freedom. Three methods of derivative reduction of such higher derivative corrections were examined and compared on several examples of single scalar field systems, at different orders in the perturbation parameter.

The methods examined are (i) derivative reduction at the equations of motion level, (ii) derivative reduction via perturbative constraints, and (iii) derivative reduction via field redefinitions. The lessons learned are summarized in the bullet points at the end of section 3. The main conclusion is that the field redefinition method, when applicable, is the most practical one, compatible with global (Lorentz) symmetries, yielding as a result a configuration space action with no genuine higher derivative corrections to a given order.

Few words are in order on why field redefinition is a legitimate method to use. When employed to eliminate higher derivative terms to certain order of interest, field redefinition necessarily involves derivatives itself which is usually not admissible. It is often pragmatically argued that in EFTs this is admissible due to pushing the problem of higher derivatives beyond the perturbative order of interest. Even though this rationale is strictly not wrong, a stronger, but more subtle argument can be made. In general, nonlocal field redefinitions are admissible if they do not change the number of degrees of freedom in the action. We can imagine such nonlocal field redefinitions that have a local derivative expansion analogous to the derivative expansion of the nonlocal correction to the action defining the EFT expansion. In particular, we can imagine a special nonlocal field redefinition whose local expansion is responsible for cancelling the genuine higher derivative terms from the EFT expansion of the action. In this sense the presence of higher derivatives in the field redefinition is not seen as fundamental, but just as a consequence of the approximation scheme. This is the reasoning behind employing field redefinitions to remove spurious degrees of freedom from the EFT expansion.

Genuine higher derivatives in EFTs are thus seen as artefacts of the approximation scheme, not as essential feature. Hence, there is no reason to look for a UV principle that would forbid their appearance in the low energy EFTs. Rather, we ought to write EFT expansions with an additional requirement - no terms in the expansion should be genuine higher derivative terms, i.e. we should not write the terms introducing new degrees of freedom. This would remedy the error we are making by using implicitly the local derivative expansion as the definition of the EFT. The examples considered in this work would suggest that EFTs should consist of all the possible corrections to the Lagrangian respecting assumed symmetries, and not introducing new degrees of freedom. In all likelihood this will not hold in general, since it is unclear that there are enough of healthy terms in a given expansion to capture all the effects. Even though field redefinitions will not generally remove all of the genuibe higher derivative terms (as suggested in [5]), there ought to be a way to capture the EFT corrections without introducing spurious degrees of freedom, but this might not be a Lagrangian (or even local) description. 
Observations about EFTs has made here bear relevance for the scalar-tensor theories often studied today. The most general scalar-tensor theories with no genuine higher derivative terms [41] go under the acronym DHOST (Degenerate-Higher-Order-Scalar-Tensor) theories. Here we suggest that, together with the most general such theories involving curvature tensors as well, they can be seen to arise as EFT corrections, ordered in the number of derivatives. However, EFT expansions can be ordered differently. Pure gravity EFT can be ordered in powers of the curvature tensors, where different kinds of non-genuine higher derivative terms appear, that are essentially nonlocal, and do not propagate linearized ghosts around maximally symmetric spacetimes $[44,45]$. Such nonlocal corrections are not addressed by the analysis in this work.

Here we have shown explicitly that the first order correction in the EFT expansion of the scalar-tensor theory is captured correctly by the most general scalar-tensor theory with at most four derivatives that does not contain extra degrees of freedom,

$$
\begin{aligned}
S= & \int d^{D} x \sqrt{-g}\left\{\frac{1}{\kappa^{2}}\left[F_{1} R-\frac{1}{2} F_{2}\left(\nabla_{\mu} \varphi\right)\left(\nabla^{\mu} \varphi\right)-F_{3}\right]+F_{4}\left(\nabla_{\mu} \varphi\right)\left(\nabla^{\mu} \varphi\right)\left(\nabla_{\nu} \varphi\right)\left(\nabla^{\nu} \varphi\right)\right. \\
& \left.+F_{5}\left(\nabla_{\mu} \varphi\right)\left(\nabla^{\mu} \varphi\right)(\square \varphi)+F_{6} G^{\mu \nu}\left(\nabla_{\mu} \varphi\right)\left(\nabla_{\nu} \varphi\right)+F_{7}\left(R^{2}-4 R_{\mu \nu} R^{\mu \nu}+R_{\mu \nu \rho \sigma} R^{\mu \nu \rho \sigma}\right)\right\},
\end{aligned}
$$

where $F_{1}-F_{7}$ are some functions of the scalar field, $F_{i}=F_{i}(\varphi)$ that in principle have an expansion in $\kappa^{2}$. Even though higher derivatives appear in this action they are not genuine higher derivatives. In $D=4$ spacetime dimensions this action propagates only one scalar and two tensor degrees of freedom. This is not the minimal action resulting from applying filed redefinitions, since $F_{5}$ and $F_{6}$ can still be set to zero, but the question we were addressing is only the removal of genuine higher derivative terms. The findings in this work agree with the findings of [5], where the same question was examined in the scalartensor theory with a shift-symmetry for the scalar. In the shift-symmetric case the last term in the action above also drops out in $D=4$ due to the Gauss-Bonnet term being a total derivative, and $F_{7}$ being just a constant. It was also found in [5] that for dimension six operators there are more higher derivative terms that cannot be removed by field redefinitions than there are possible non-genuine higher derivative terms, suggesting that in general field redefinitions cannot remove all spurious degrees of freedom from the EFT expansion. However, it is not certain that all non-genuine higher derivative dimension six terms are known, and hence it is not certain exactly how many genuine higher derivative terms of dimension six cannot be removed.

General proofs of the statements made here about the absence of genuine higher derivative corrections in EFTs would be very powerful, as the authors of [4] point out. It should be pointed out that the difficult part in any such proof at higher orders than ones considered here is classifying the non-genuine higher derivative corrections which need not be removed. This cannot be done by simply counting the number of derivatives appearing in the action, but rather one often has to resort to canonical formalism to be able to count the number of degrees of freedom correctly [41] . 


\section{Acknowledgments}

The author is indebted to Tomislav Prokopec for useful suggestions. This work was supported by the grant 2014/14/E/ST9/00152 of the Polish National Science Centre (NCN).

Open Access. This article is distributed under the terms of the Creative Commons Attribution License (CC-BY 4.0), which permits any use, distribution and reproduction in any medium, provided the original author(s) and source are credited.

\section{References}

[1] J.D. Wells, Effective theories in physics: From planetary orbits to elementary particle masses, Springer, (2012), [https://doi.org/10.1007/978-3-642-34892-1].

[2] C.P. Burgess, Introduction to Effective Field Theory, Ann. Rev. Nucl. Part. Sci. 57 (2007) 329 [hep-th/0701053] [inSPIRE].

[3] J.F. Donoghue, Introduction to the effective field theory description of gravity, gr-qc/9512024 [INSPIRE].

[4] C.P. Burgess and M. Williams, Who You Gonna Call? Runaway Ghosts, Higher Derivatives and Time-Dependence in EFTs, JHEP 08 (2014) 074 [arXiv:1404.2236] [INSPIRE].

[5] A.R. Solomon and M. Trodden, Higher-derivative operators and effective field theory for general scalar-tensor theories, arXiv:1709.09695 [INSPIRE].

[6] M. Ostrogradsky, Mémoires sur les équations différentielles, relatives au problème des isopérimètres, Mem. Acad. St. Petersbourg 6 (1850) 385 [INSPIRE].

[7] R.P. Woodard, Ostrogradsky's theorem on Hamiltonian instability, Scholarpedia 10 (2015) 32243 [arXiv: 1506. 02210] [INSPIRE].

[8] G.W. Horndeski, Second-order scalar-tensor field equations in a four-dimensional space, Int. J. Theor. Phys. 10 (1974) 363 [INSPIRE].

[9] A. Nicolis, R. Rattazzi and E. Trincherini, The Galileon as a local modification of gravity, Phys. Rev. D 79 (2009) 064036 [arXiv:0811.2197] [INSPIRE].

[10] C. Deffayet, G. Esposito-Farese and A. Vikman, Covariant Galileon, Phys. Rev. D 79 (2009) 084003 [arXiv: 0901.1314] [INSPIRE].

[11] C. Deffayet, S. Deser and G. Esposito-Farese, Generalized Galileons: All scalar models whose curved background extensions maintain second-order field equations and stress-tensors, Phys. Rev. D 80 (2009) 064015 [arXiv: 0906.1967] [INSPIRE].

[12] J. Gleyzes, D. Langlois, F. Piazza and F. Vernizzi, Healthy theories beyond Horndeski, Phys. Rev. Lett. 114 (2015) 211101 [arXiv:1404.6495] [InSPIRE].

[13] J. Gleyzes, D. Langlois, F. Piazza and F. Vernizzi, Exploring gravitational theories beyond Horndeski, JCAP 02 (2015) 018 [arXiv: 1408.1952] [INSPIRE].

[14] D. Langlois and K. Noui, Degenerate higher derivative theories beyond Horndeski: evading the Ostrogradski instability, JCAP 02 (2016) 034 [arXiv:1510.06930] [INSPIRE].

[15] M. Crisostomi, K. Koyama and G. Tasinato, Extended Scalar-Tensor Theories of Gravity, JCAP 04 (2016) 044 [arXiv: 1602.03119] [INSPIRE]. 
[16] J. Ben Achour, M. Crisostomi, K. Koyama, D. Langlois, K. Noui and G. Tasinato, Degenerate higher order scalar-tensor theories beyond Horndeski up to cubic order, JHEP 12 (2016) 100 [arXiv: 1608.08135] [INSPIRE].

[17] G. Gubitosi, F. Piazza and F. Vernizzi, The Effective Field Theory of Dark Energy, JCAP 02 (2013) 032 [arXiv: 1210.0201] [INSPIRE].

[18] S. Tsujikawa, The effective field theory of inflation/dark energy and the Horndeski theory, Lect. Notes Phys. 892 (2015) 97 [arXiv:1404.2684] [INSPIRE].

[19] J.D. Jackson, Classical Electrodynamics, Wiley (1962).

[20] J.Z. Simon, Higher Derivative Lagrangians, Nonlocality, Problems and Solutions, Phys. Rev. D 41 (1990) 3720 [INSPIRE].

[21] L. Parker and J.Z. Simon, Einstein equation with quantum corrections reduced to second order, Phys. Rev. D 47 (1993) 1339 [gr-qc/9211002] [INSPIRE].

[22] F.D. Mazzitelli, Higher derivatives and renormalization in quantum cosmology, Phys. Rev. D 45 (1992) 2814 [INSPIRE].

[23] S. Cremonini, J.T. Liu and P. Szepietowski, Higher Derivative Corrections to R-charged Black Holes: Boundary Counterterms and the Mass-Charge Relation, JHEP 03 (2010) 042 [arXiv: 0910.5159] [INSPIRE].

[24] G. Goon, Heavy Fields and Gravity, JHEP 01 (2017) 045 [Erratum ibid. 03 (2017) 161] [arXiv: 1611.02705] [INSPIRE].

[25] J. Berges, Introduction to nonequilibrium quantum field theory, AIP Conf. Proc. 739 (2005) 3 [hep-ph/0409233] [INSPIRE].

[26] T. Prokopec and J. Weenink, Uniqueness of the gauge invariant action for cosmological perturbations, JCAP 12 (2012) 031 [arXiv:1209.1701] [INSPIRE].

[27] T. Prokopec and J. Weenink, Frame independent cosmological perturbations, JCAP 09 (2013) 027 [arXiv: 1304.6737] [INSPIRE].

[28] G. Domènech and M. Sasaki, Hamiltonian approach to 2nd order gauge invariant cosmological perturbations, arXiv:1709.09804 [INSPIRE].

[29] S.P. Miao and R.P. Woodard, Issues Concerning Loop Corrections to the Primordial Power Spectra, JCAP 07 (2012) 008 [arXiv:1204.1784] [INSPIRE].

[30] D. Langlois, Hamiltonian formalism and gauge invariance for linear perturbations in inflation, Class. Quant. Grav. 11 (1994) 389 [INSPIRE].

[31] N.D. Birrell and P.C.W. Davies, Quantum Fields in Curved Space, Cambridge University Press, (2012), [https://doi.org/10.1017/CBO9780511622632]

[32] D.M. Gitman and I.V. Tyutin, Quantization of Fields with Constraints, Springer (1990) [https://doi.org/10.1007/978-3-642-83938-2_1].

[33] P.A.M. Dirac Lectures on Quantum Mechanics, Belfer Graduate School of Science, monograph series, Dover Publications, (2001).

[34] X. Jaen, J. Llosa and A. Molina, A reduction of order two for infinite order lagrangians, Phys. Rev. D 34 (1986) 2302 [INSPIRE].

[35] D.A. Eliezer and R.P. Woodard, The Problem of Nonlocality in String Theory, Nucl. Phys. B 325 (1989) 389 [INSPIRE]. 
[36] M. Crisostomi, R. Klein and D. Roest, Higher Derivative Field Theories: Degeneracy Conditions and Classes, JHEP 06 (2017) 124 [arXiv: 1703.01623] [INSPIRE].

[37] D. Lovelock, The Einstein tensor and its generalizations, J. Math. Phys. 12 (1971) 498 [INSPIRE].

[38] C. Lanczos, A Remarkable property of the Riemann-Christoffel tensor in four dimensions, Annals Math. 39 (1938) 842.

[39] S. Nojiri, S.D. Odintsov and M. Sasaki, Gauss-Bonnet dark energy, Phys. Rev. D 71 (2005) 123509 [hep-th/0504052] [INSPIRE].

[40] D. Langlois, Degenerate Higher-Order Scalar-Tensor (DHOST) theories, arXiv:1707.03625 [INSPIRE].

[41] D. Langlois and K. Noui, Hamiltonian analysis of higher derivative scalar-tensor theories, JCAP 07 (2016) 016 [arXiv: 1512.06820] [INSPIRE].

[42] G. 't Hooft and M.J.G. Veltman, One loop divergencies in the theory of gravitation, Ann. Inst. H. Poincare Phys. Theor. A 20 (1974) 69.

[43] S. Weinberg, Effective Field Theory for Inflation, Phys. Rev. D 77 (2008) 123541 [arXiv: 0804.4291] [INSPIRE].

[44] T. Biswas, A.S. Koshelev and A. Mazumdar, Consistent higher derivative gravitational theories with stable de Sitter and anti-de Sitter backgrounds, Phys. Rev. D 95 (2017) 043533 [arXiv: 1606.01250] [INSPIRE].

[45] T. Biswas, A.S. Koshelev and A. Mazumdar, Gravitational theories with stable (anti-)de Sitter backgrounds, Fundam. Theor. Phys. 183 (2016) 97 [arXiv:1602.08475] [INSPIRE].

[46] R. Gwyn, G.A. Palma, M. Sakellariadou and S. Sypsas, Effective field theory of weakly coupled inflationary models, JCAP 04 (2013) 004 [arXiv: 1210.3020] [INSPIRE].

[47] J.-O. Gong, M.-S. Seo and S. Sypsas, Higher derivatives and power spectrum in effective single field inflation, JCAP 03 (2015) 009 [arXiv: 1407.8268] [INSPIRE].

[48] E. Castillo, B. Koch and G. Palma, On the integration of fields and quanta in time dependent backgrounds, JHEP 05 (2014) 111 [arXiv: 1312.3338] [INSPIRE]. 